www.nature.com/pj

\title{
Synthesis and properties of soft nanocomposite materials with novel organic/inorganic network structures
}

\author{
Kazutoshi Haraguchi
}

We have fabricated new types of polymer hydrogels and polymer nanocomposites, that is, nanocomposite gels (NC gels) and soft polymer nanocomposites (M-NCs), with novel organic/inorganic network structures. Both NC gels and M-NCs were synthesized by in situ free-radical polymerization in the presence of exfoliated clay platelets in aqueous systems and were obtained in various forms and sizes with a wide range of clay contents. Here, disk-like inorganic clay nanoparticles function as multifunctional crosslinkers to form new types of network systems. NC gels have extraordinary optical, mechanical and swelling/deswelling properties, as well as a number of new characteristics relating to optical anisotropy, polymer/clay morphology, biocompatibility, stimuli-sensitive surfaces, micropatterning and so on. The M-NCs also exhibit dramatic improvements in optical and mechanical properties including ultrahigh reversible extensibility and well-defined yielding behavior, despite their high clay contents. Thus, the serious disadvantages (intractability, mechanical fragility, optical turbidity, poor processing ability, low stimulus sensitivity and so on) associated with the conventional, chemically crosslinked polymeric materials were overcome in NC gels and M-NCs. Polymer Journal (2011) 43, 223-241; doi:10.1038/pj.2010.141; published online 19 January 2011

Keywords: clay; hydrogel; mechanical properties; nanocomposite; network

\section{INTRODUCTION}

Polymer nanocomposites (P-NCs), composed of organic polymer and inorganic nanoparticles, have been widely investigated in the last three decades to develop new, value-added polymeric materials based on existing polymers. ${ }^{1-4}$ To date, many P-NCs consisting of various polymers and inorganic nanoparticles (for example, silica, silsesquioxane, titania, clay, carbon nanotubes) have been developed by using sol-gel reactions of metal alkoxides or organic premodification of nanoparticles. ${ }^{1-5}$ The resulting P-NCs show significant improvements in some properties, such as modulus, heat-distortion temperature, hardness, gas impermeability and so on. However, the P-NCs developed so far have encountered inherent difficulties in preparation and processing as the inorganic content increases. In the case of polymer/ clay nanocomposites, in general, only a few weight percent of clay can actually be incorporated into NCs ( $<10 \mathrm{wt} \%$, at the most) in the form of organic modified clay. ${ }^{2,4,5}$ Further increases in clay content often cause structural inhomogeneities because of inadequate dispersion or irregular aggregation of the clay, and always result in disadvantageous optical and mechanical properties and processability.

To overcome these limitations, we extend the concept of 'organic/ inorganic nanocomposites' to the field of soft materials, such as 'polymer hydrogels' and 'soft polymers,' under the strategy of fabricating novel organic/inorganic structures. In the present article, we give an overview of the development of two types of soft nanocomposites, that is, nanocomposite gels ${ }^{6-10}$ and soft polymer nanocomposites, ${ }^{11,12}$ with unique organic/inorganic network structures, which break through the previous limitations and exhibit outstanding new characteristics as well as excellent optical and mechanical properties.

\section{NANOCOMPOSITE GELS}

Disadvantages of polymer hydrogels

Polymer hydrogels, which consist of three-dimensional (3D) polymer networks and large amounts of water, are soft, wet materials with the characteristics of both solids (well-defined shapes) and liquids (free water in most cases). The late Professor Tanaka described the scientific interest of hydrogels in connection with certain physical critical phenomena and bio-related materials. ${ }^{13}$ When a polymer with stimulus sensitivity is used as a network constituent, a stimulus-sensitive polymer hydrogel is obtained. In particular, poly $(N$-isopropylacrylamide) (PNIPA), which undergoes a thermoresponsive coil-to-globule transition at a lower critical solution temperature (LCST $\approx 32^{\circ} \mathrm{C}$ ) in aqeous media, ${ }^{14}$ is a typical example of a smart gel. To date, extensive studies have been conducted into the feasibility of using PNIPA hydrogels in various systems such as artificial insulin-control systems, ${ }^{15}$ efficient bioseparation devices, ${ }^{16}$ drug delivery systems ${ }^{17}$ and biotechnological and tissue engineering devices. ${ }^{18,19}$

Among the various types of polymer hydrogels, 'chemically crosslinked polymer hydrogels' have been widely used in academic studies 
as well as for practical applications, such as soft contact lenses and superabsorbent polymers, because their network composition and degree of crosslinking can be easily controlled. PNIPA hydrogels described above have also been prepared by chemical crosslinking using an organic crosslinker. It is known that chemically crosslinked polymer hydrogels (hereinafter abbreviated as OR gels, as they are prepared using organic crosslinkers) have several serious limitations because of their network structure, which consists of a random arrangement of a large number of chemical crosslinks (Figure 1a(i)). ${ }^{6,8}$ Here, an increase in the crosslink density $(v)$ is accompanied by a decrease in the intercrosslink molecular weight $\left(M_{\mathrm{c}}\right), v \propto M_{\mathrm{c}}{ }^{-1}$. Furthermore, as the crosslinking reaction cannot occur at regularly separated positions, $M_{\mathrm{c}}$ is always characterized by a broad distribution of chain lengths between crosslinking points. Furthermore, structural inhomogeneity due to the heterogeneous aggregation of crosslinking points often occurs when the crosslinker concentration is high. ${ }^{20}$ Therefore, OR gels have some serious disadvantages: (1) optical opacity at a high $v$; (2) poor mechanical strength and brittleness, irrespective of $v$; (3) low degree of swelling and slow deswelling rate due to restrictions in the movement of polymer chains by chemical crosslinks, even at the moderate crosslinker concentrations commonly used. ${ }^{6,20}$

Figure 1a shows tensile stress-strain curves for OR gels prepared with different concentrations of organic crosslinker $\left(N, N^{\prime}\right.$-methylenebis (acrylamide) (BIS)), $C_{\mathrm{BIS}}$ in mol\%, over a wide range $\left(C_{\mathrm{BIS}}=0.001-3\right){ }^{8}$ Also included are data for an aqueous solution of PNIPA (linear polymer (LR)), prepared under the same conditions as OR gel except for the absence of a crosslinker, which formed a very viscous, sticky, gel-like material. The LR could be irreversibly elongated extensively (near to or more than $3000 \%)$ at very low stress $(<4 \mathrm{kPa})$. This is because PNIPA has a high molecular weight and forms a lightly, self-crosslinked network, including topological entanglements. ${ }^{21}$

Figure 1a also shows that the initial modulus $(E)$ gradually increases with increasing $C_{\mathrm{BIS}}$, with a simultaneous rapid decrease in elongation at break $\left(\varepsilon_{\mathrm{b}}\right)$. Consequently, the ultimate tensile strength $(\sigma)$ remained low $(\sim 10 \mathrm{kPa})$ throughout the whole range of $C_{\mathrm{BIS}}(\propto v)$. These weak, brittle properties of OR gels are attributed to their network structure. As shown in Figure 1a(ii), on unidirectional extension, polymer chains with a broad distribution of $M_{\mathrm{c}}$ are successively broken because of stress localization in the shorter chains present at any instant. Thus, the chemically crosslinked network structure is a principal factor responsible for the fragility of OR gels. Therefore, to dramatically enhance the mechanical properties of these gels, it is necessary to fabricate new network structures, preferably by using conditions in which $v$ and $M_{\mathrm{c}}$ can be independently controlled.

Recently, remarkable improvements have been made to the mechanical properties of polymeric hydrogels by creating new types of network structures using different strategies, such as networks with sliding crosslinks (slide-ring gels), ${ }^{22}$ organic/inorganic networks (NC gels), ${ }^{6}$ interpenetrating networks (double-network gels), ${ }^{23}$ selfassembled networks (macromolecular microsphere composite gels) ${ }^{24}$ and networks with the same $M_{\mathrm{c}}$ (tetra-poly(ethylene glycol) (PEG) gels). ${ }^{25}$ Among these hydrogels, nanocomposite gels (abbreviated as NC gels) consisting of organic (polymer) and inorganic clay overcame all the problems associated with OR gels simultaneously, $6,7,20,26$ and achieved the best mechanical properties together with excellent optical, swelling and stimuli-sensitive properties.

\section{Synthesis of NC gels}

NC gels were prepared by in situ free-radical polymerization of watersoluble monomers containing amide groups, such as $N$-isopropylacrylamide (NIPA), N,N-dimethylacrylamide and acrylamide, in the a
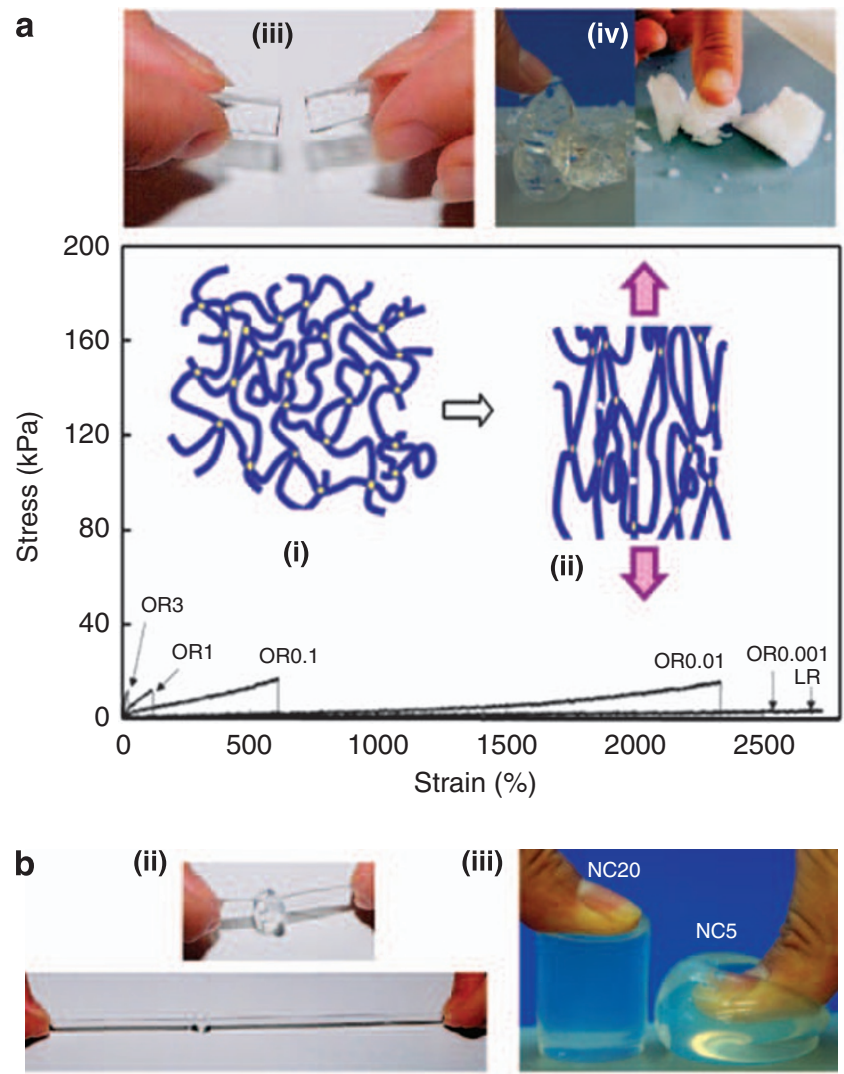

(iii)
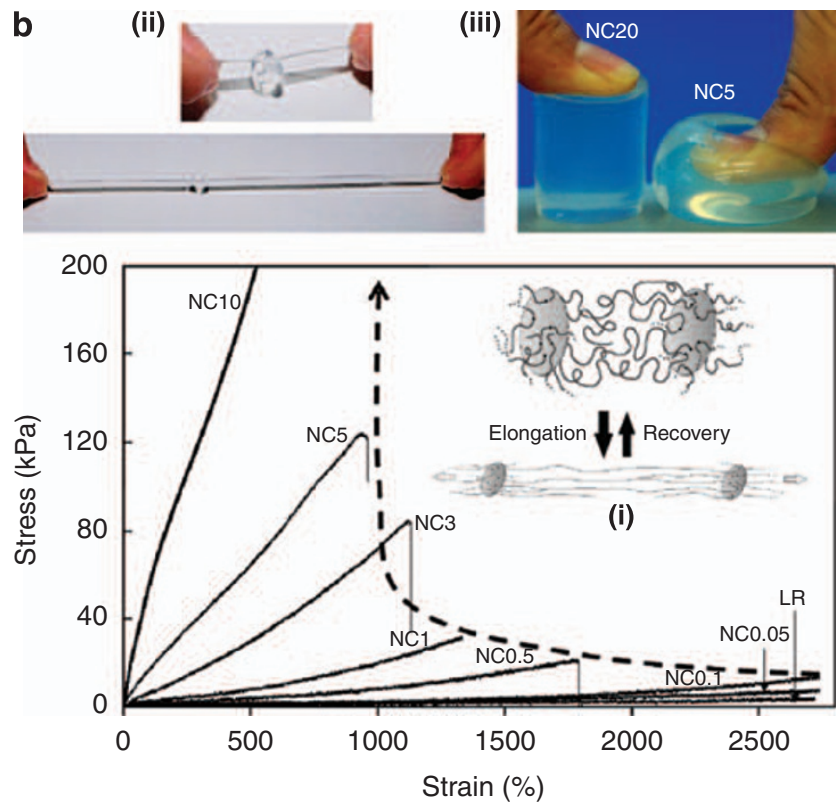

Figure 1 (a) Tensile stress-strain curves of $\mathrm{N}$-OR gels with different $C_{\mathrm{BIS}}$ $(0.001-3)$ and linear polymer (LR; $\left.C_{\mathrm{BIS}}=0\right) .6,8,20$ (i) Schematic representation of the network of chemically crosslinked OR gel. (ii) Rupture of crosslinked chains on unidirectional extension. (iii), (iv) OR1 gel fractured at small stress by elongation and compression. (b) Tensile stress-strain curves of N-NC gels with different $C_{\text {clay }}(0.05-10)$ and LR. ${ }^{6-8,20}$ (i) A schematic representation of the unit structure of an organic/inorganic network. (ii) A knotted and stretched NC5 gel. (iii) Compression of NC5 and NC20 gel.

presence of inorganic clay that has been exfoliated and uniformly dispersed in aqueous media. ${ }^{6,7,20,26,27}$ A variety of clay minerals with layered crystal structures and good water swellability can be used as the inorganic component; examples are the smectite-group clays (hectorite, saponite, montmorillonite and so on) and mica-group clays (synthetic fluorine mica). Among these, the synthetic hectorite 'Laponite XLG' (Rockwood Ltd, UK; $\left[\mathrm{Mg}_{5.34} \mathrm{Li}_{0.66} \mathrm{Si}_{8} \mathrm{O}_{20}(\mathrm{OH})_{4}\right] \mathrm{Na}_{0.66}$; layer size: $30 \mathrm{~nm}$ (diameter) $\times 1 \mathrm{~nm}$ (thickness); cation-exchange 
a

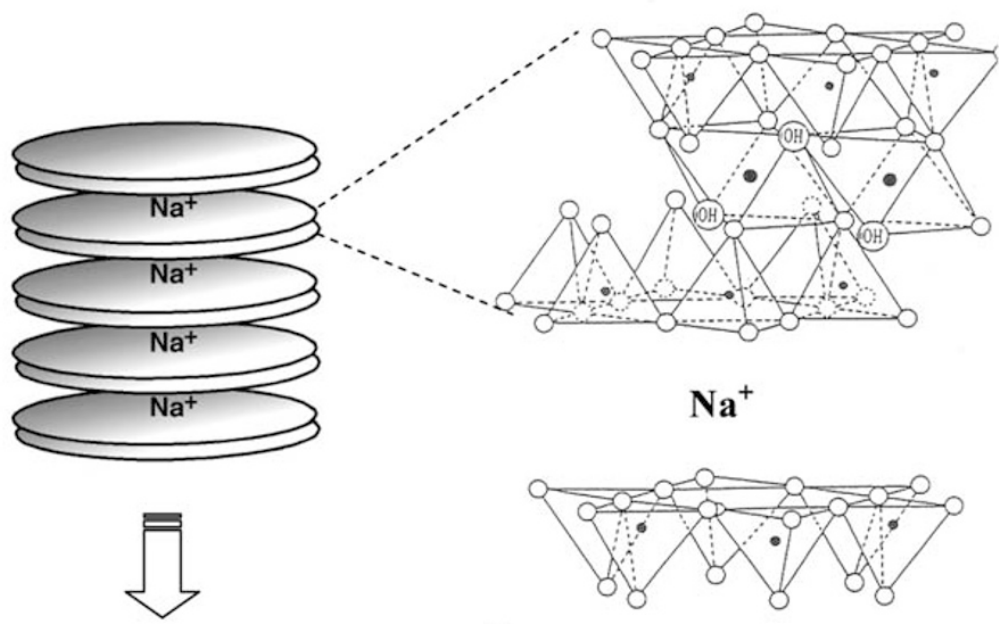

b

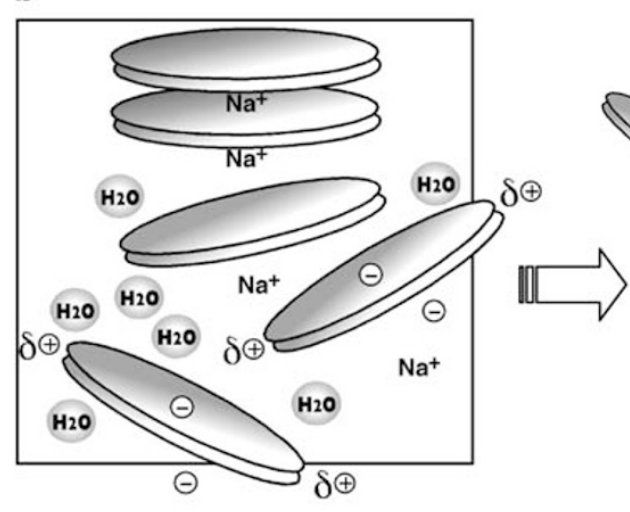

C

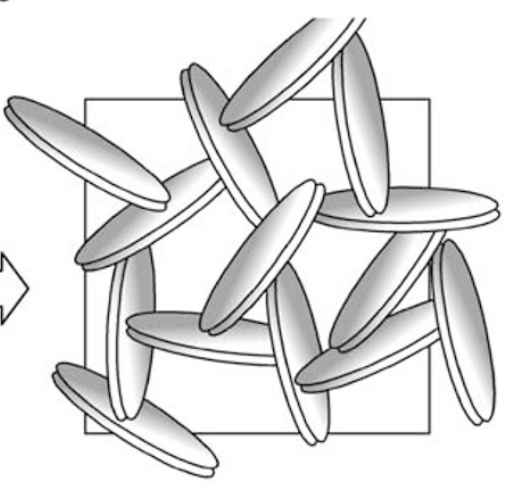

"House of Cards" structure

Figure 2 (a) Structure of hectorite, (b) exfoliation in aqueous media, (c) house-of-card structure. ${ }^{31}$

capacity: 104 mequiv per $100 \mathrm{~g}),{ }^{26,28}$ which has a 2:1 layered structure (Figure 2), was most effective because of its high degree of swellability and exfoliation, high purity, sufficiently small platelet size and good interaction with PNIPA. In the present review, inorganic clay refers to Laponite XLG, unless otherwise stated. For example, to synthesize an N-NC3 gel (see the nomenclature below), a transparent aqueous solution consisting of water $(20 \mathrm{ml})$, clay (XLG: $0.457 \mathrm{~g})$, monomer (NIPA: $2.26 \mathrm{~g}$ ), initiator (potassium persulfate: $0.02 \mathrm{~g}$ ) and accelerator $\left(N, N, N^{\prime}, N^{\prime}\right.$-tetramethylenediamine: $\left.16 \mu \mathrm{l}\right)$ was prepared at ice bath temperature and then the temperature was increased to $20^{\circ} \mathrm{C}$ to initiate in situ free-radical polymerization.

Consequently, structurally uniform, transparent hydrogels (NC gels) are obtained without syneresis or phase separation. Polymerization yields are almost $100 \%$, regardless of the NC gel composition. The compositions of NC gels can, therefore, be precisely controlled over a wide range by altering those of the initial reaction solutions. It should be noted that, for the synthesis of NC gels, organic crosslinkers, which afford chemically crosslinked networks, are not required. More specifically, such crosslinkers should not be used in conjunction with clay. When two crosslinkers, clay and BIS, are used together $\left(C_{\text {clay }}=5\right.$ and $C_{\mathrm{BIS}}=1$ ), the resulting hydrogel (NC5-OR1 gel) becomes weak and brittle on elongation, similar to an OR1 gel. ${ }^{29}$

The methods used to prepare NC gels are simple and versatile; that is, injecting reaction solutions into closed vessels followed by polymerization at ambient temperature. Hence, NC gels can be readily formed in various shapes and sizes, such as films, sheets, rods, spheres and hollow tubes, as shown in Figure $3 .{ }^{8,9} \mathrm{NC}$ gels can also be prepared by photoinitiated free-radical polymerization using very low concentrations (for example, $0.1 \mathrm{wt} \%$ relative to the monomer) of a hydrophobic photoinitiator in aqueous systems. ${ }^{30}$ Futhermore, the other type of NC gel, that is, tetra-TEG-based NC gels, with good biocompatibility can be prepared by incorporating clay nanoparticles into the tetra-poly(ethylene glycol) network. ${ }^{10}$

The nomenclature codes used to identify NC and OR gels are based on the monomer used (N- and D- for NIPA and N,N-dimethylacrylamide, respectively) and on the concentrations of clay and monomer relative to water; thus, $\mathrm{N}-\mathrm{NC} n-\mathrm{M} m$ indicates an $\mathrm{NC}$ gel prepared using $n \times 10^{-2} \mathrm{~mol}$ of clay and $m \mathrm{~mol}$ of NIPA in $11 \mathrm{H}_{2} \mathrm{O}$, and D-OR $n^{\prime}$ indicates an OR gel prepared using $n^{\prime} \mathrm{mol} \%$ of BIS relative to monomer and $1 \mathrm{M}$ of $N, N$-dimethylacrylamide. When $m$ is 1 , the last symbol (-M1) is often omitted for simplicity. Furthermore, the initial symbol (N- or D-) is sometimes omitted in NC and OR gels wherein there is no possibility of confusion. The clay, polymer and BIS contents of NC and OR gels $\left(C_{\text {clay }}, C_{\mathrm{p}}\right.$ and $\left.C_{\mathrm{BIS}}\right)$ are expressed using simplified numerical values of $n, m$ and $n^{\prime}$, respectively.

\section{ORGANIC/INORGANIC NETWORK STRUCTURES}

Formation of networks with clay

Gel formation, that is, formation of polymer networks with inorganic clay (in the absence of an organic crosslinker), was confirmed by their swelling and dynamic mechanical properties. In dynanic mechanical measurements at $22^{\circ} \mathrm{C},{ }^{8}$ it was observed for D-NC5 gel that the storage modulus $\left(G^{\prime}\right)$ is always greater than the loss modulus $\left(G^{\prime \prime}\right)$ in the frequency range $10^{-1} \sim 10^{2} \mathrm{rad} \mathrm{s}^{-1}$, and that $G^{\prime}$ and $G^{\prime \prime}$ change 

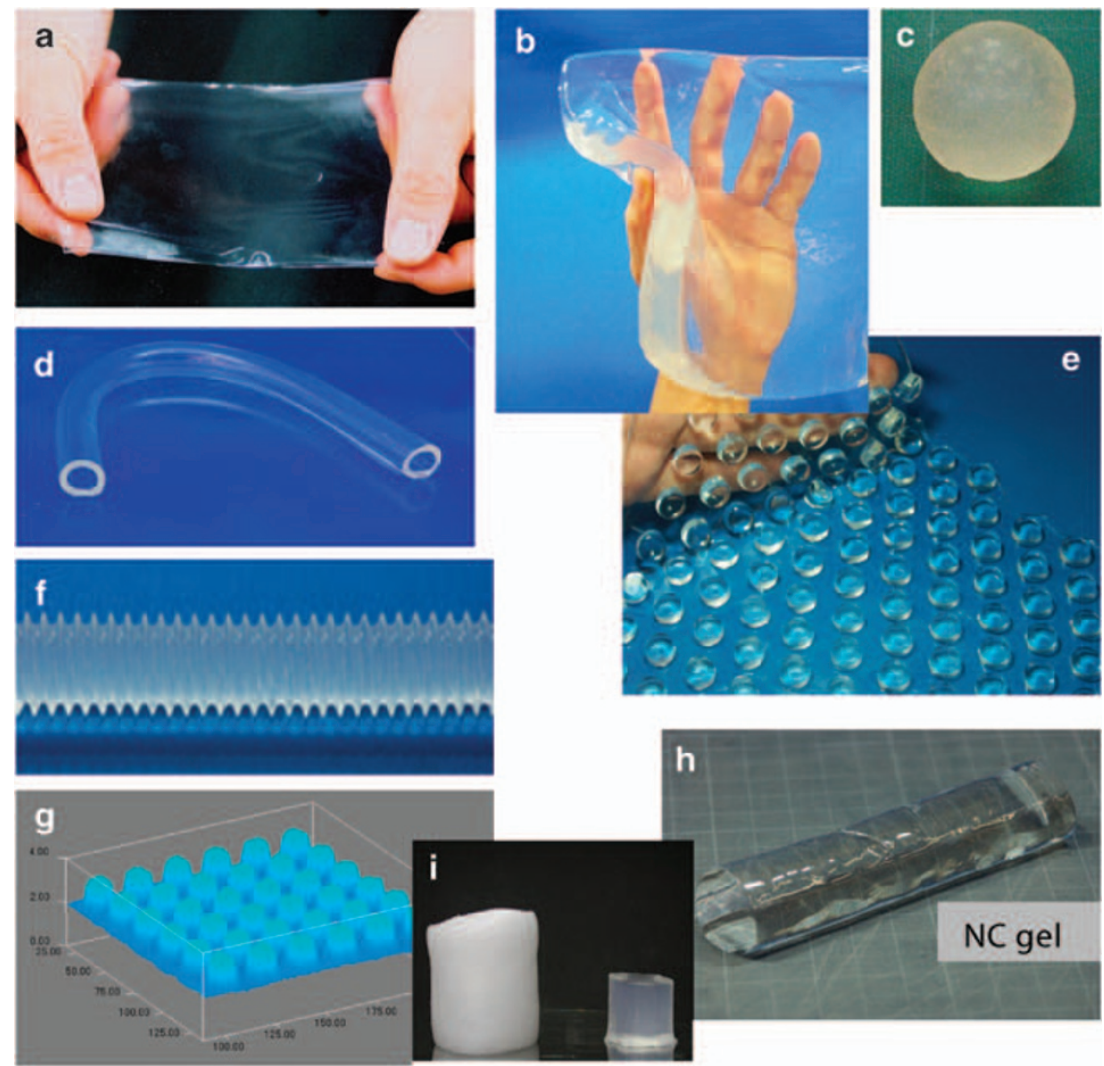

Figure 3 NC gels with various shapes and forms. (a) Thin film, (b) sheet, (c) sphere, (d) hollow tube, (e) sheet with uneven surface, (f) bellows. (g) Laserscanning confocal microscopy 3D images of the surface pattern $(10 \mu \mathrm{m}$ square dot holes with $10 \mu \mathrm{m}$ intervals) of NC gel films prepared by direct replica molding. (h) NC gels containing 99.8 wt $\% \mathrm{H}_{2} \mathrm{O}$. (i) Porous NC gel. $8,9,68$

little with frequency. The constantly high $G^{\prime}\left(>G^{\prime \prime}\right)$ indicates that viscoelastic relaxation does not occur on this time scale. These observations are consistent with the dynamic viscoelastic properties that characterize hydrogels with 3D networks. In addition, network formation in NC gels was confirmed by their rubbery nature, such as reversible large deformation and recovery in stretching and compression tests, as shown in a later section.

Network formation in NC gels was further ascertained by swelling measurements. NC gels do not dissolve in water but swell until they reach an equilibrium state, with no free linear polymer chains or free clay particles separating from the network. ${ }^{20,26}$ These results indicate that NC gels form 3D networks in which all the polymer chains and clay particles are incorporated. In general, the degree of equilibrium swelling of a hydrogel is inversely proportional to $v$. In the case of NC gels, the degree of swelling changes with the gel composition; that is, degree of equilibrium swelling tends to decrease with increasing $C_{\text {clay }}$ and $C_{\mathrm{p}}{ }^{26}$ This indicates that the networks in NC gels are formed by crosslinking polymer chains by clay in a specific manner.

\section{Polymer/clay network structure}

Various analytical studies (transmission electron microscopy, thermogravimetry, X-ray diffraction (XRD), differential-scanning calorimetry, Fourier-transform infrared spectroscopy for dried NC gels; dynamic light scattering and small-angle neutron scattering (SANS) for NC gels) revealed the following structural aspects of NC gels: ${ }^{6,20,26,31-36}$ (1) Disk-like inorganic clay nanoparticles $(30 \mathrm{~nm}$ $\phi \times 1 \mathrm{~nm})$, resulting from exfoliation of the layered clay mineral (hectorite), are uniformly dispersed in a polymer matrix (XRD, transmission electron microscopy: Figure 4c (dried N-NC5 gel)). (2) Flexible polymer chains with the same $T_{\mathrm{g}}$ as that of the LR exist in NC gels (differential-scanning calorimetry). (3) There is no distinct difference between the absorptions of PNIPA and clay in the dried NC gels and those of pure PNIPA or clay (Fourier-transform infrared spectroscopy). This is probably because, in the dried state, PNIPA shows strong hydrogen bonding, which prevents hydrogen bonding with clay from being clearly identified in dried NC gels.

On the basis of the analytical data obtained and the excellent optical, mechanical and swelling/deswelling properties of NC gels, it was concluded that NC gels possess a unique organic (polymer)/

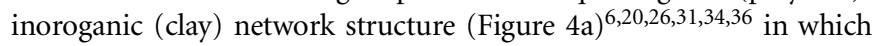
exfoliated clay nanoparticles (uniformly dispersed in an aqueous medium) are interlinked by a number of flexible polymer chains. Here, $D_{\mathrm{ic}}$ is the interparticle distance between exfoliated clay sheets. In the network, various types of polymer chains, such as grafts with free chain ends, looped chains and topologically crosslinked chains may be present in addition to the crosslinking chains. Interaction between polymers and clay nanoparticles is ascribed to non-covalent bonds, probably hydrogen bonds between the amide side groups on the polymer and the surface of the clay ( $\mathrm{SiOH}, \mathrm{Si}-\mathrm{O}-\mathrm{Si}$ units).

In the polymer/clay network structures, it was considered that a number of polymer chains interact with a single clay platelet and that each polymer chain may interact with the clay surface at multiple points. ${ }^{6,20,26,31}$ In other words, the exfoliated clay platelets function as multifunctional crosslinkers, and hence the polymer chains in NC gels are crosslinked by planar series of crosslinks. These results were confirmed by the molecular characteristics of PNIPA separated from 


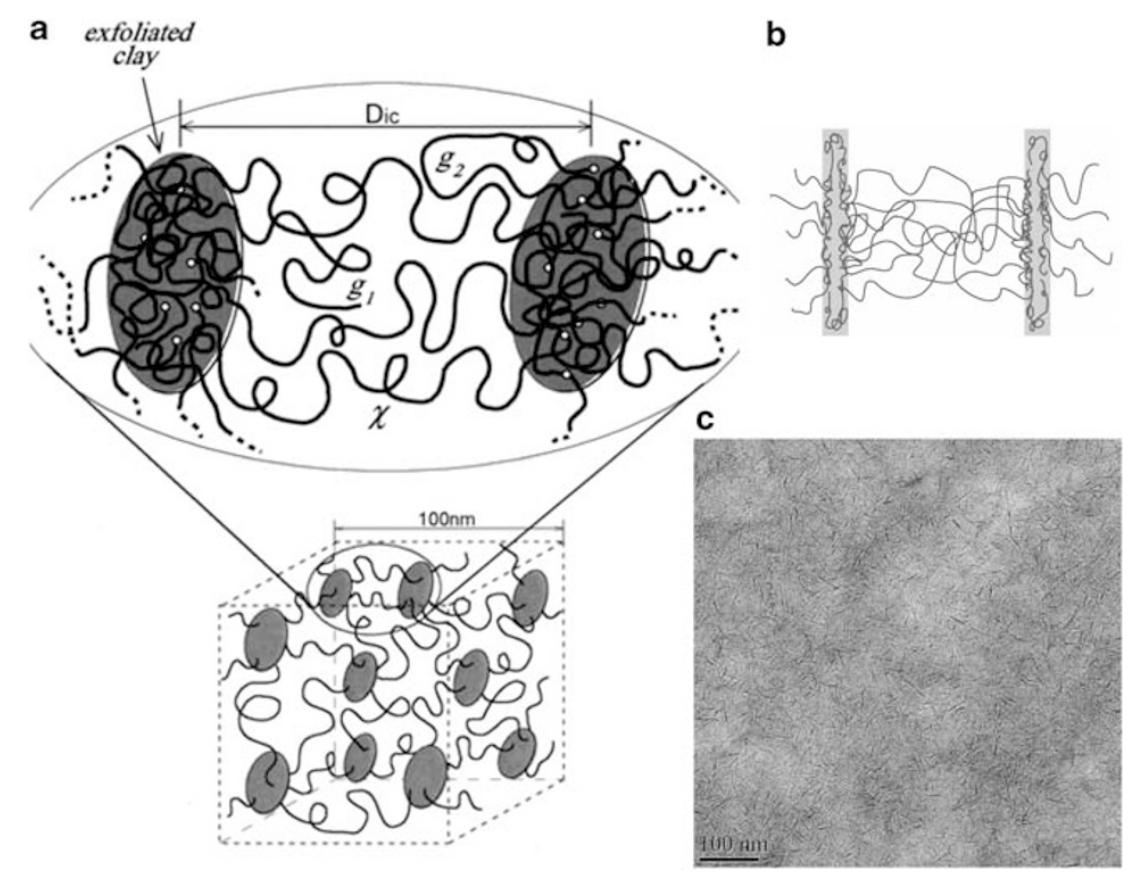

Figure 4 (a) Schematic representation of the structural model with organic (polymer)/inorganic (clay) networks in the nanocomposite gels (NC gel). $D_{\text {ic }}$ is interparticle distance of exfoliated clay sheets. $\chi, g_{1}$ and $g_{2}$ represent crosslinked chain, grafted chain and looped chain, respectively. In the model, only a small number of polymer chains are depicted for simplicity. $6,20,36$ (b) Decorated clay platelet model sandwiched by polymer layer. ${ }^{34}$ (c) Transmission electron micrographs of dried NC5 gel. The scale bar indicates $100 \mathrm{~nm} .^{20}$

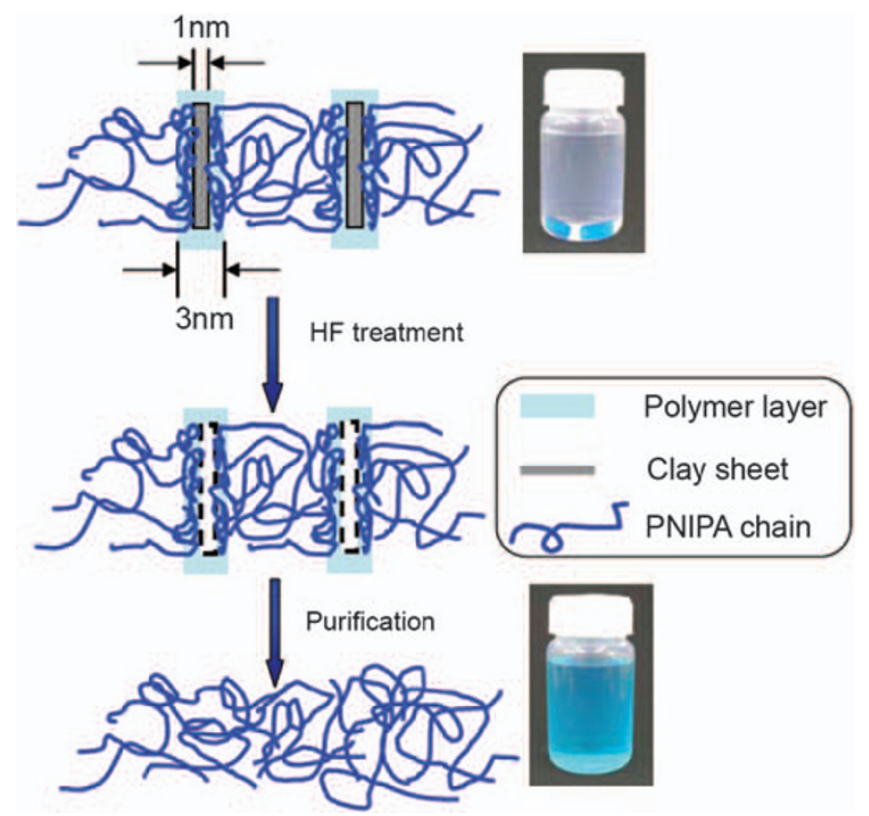

Figure 5 Schematic representation of the process of separation of poly $(N$ isopropylacrylamide) (PNIPA) from nanocomposite gels through decomposition of the clay by hydrofluoric acid (HF) treatment. ${ }^{37}$

NC gels and contrast-variation SANS measurements. By successful separation of PNIPA from the N-NC gel, without damage, by decomposing the clay in the network using hydrofluoric acid (Figure 5), it became clear that clay platelets have an important role in preventing the formation of self-crosslinked PNIPA networks, and that PNIPA in N-NC gels has a high molecular weight $\left(M_{\mathrm{w}}=5.6 \times 10^{6} \mathrm{~g} \mathrm{~mol}^{-1}\right)$, almost regardless of $C_{\text {clay }}(1-25)$, indicating that a single polymer chain repeatedly links neighboring clay sheets. ${ }^{37}$ In contrast-variation SANS measurements, it was shown that the polymer chains aggregate to form $1 \mathrm{~nm}$-thick layers on the clay surface (Figure $4 \mathrm{~b}) .^{34}$

The number of crosslinked polymer chains per unit volume of NC gel, $N^{*}$, can be estimated by Equation (1) on the basis of the kinetic theory of rubber elasticity. ${ }^{38}$

$$
F=\Phi N^{*} k_{B} T\left\{\alpha-(1 / \alpha)^{2}\right\}
$$

Here, $F$ is the force per unit original (undeformed) cross-sectional area of the swollen network, $\Phi$ is a front factor $(=1), \alpha$ is the elongation ratio and $k_{\mathrm{B}}, T$ are Boltzmann's constant and the absolute temperature, respectively. From the tensile stress-strain curves and equation (1), $N^{*}$ and the number of crosslinked chains per clay sheet were calculated for NC gels with different $C_{\text {clay }}$ values. ${ }^{26}$ The results revealed that neighboring clay sheets in $\mathrm{NC}$ gels are linked by at least tens of flexible polymer chains (a few tens to more than 100).

The effective crosslink densities $\left(v_{\mathrm{e}}\right)$ of NC gels were calculated using degree of equilibrium swelling, according to the Flory-Rehner theory. ${ }^{39}$ Here, although an $\mathrm{NC}$ gel is a kind of ionic polymer gel containing ionic clay platelets, the effect of clay on the swelling was totally different from that of usual organic ionic groups. In NC gels, the swelling decreased with increasing $C_{\text {clay }}{ }^{20,26}$ although normal ionic polymer gels exhibit increased swelling with increasing concentrations of ionic groups. This is because clay platelets function as effective crosslinkers. Therefore, to calculate $v_{\mathrm{e}}$ for an NC gel, equation (2), which corresponds to an affine network model for non-ionic gel systems, was used to simplify the condition. ${ }^{40}$

$$
\phi+\ln (1-\phi)+\chi \phi^{2}=-V_{\mathrm{s}} v_{\mathrm{e}}\left[\left(\frac{\phi}{\phi_{0}}\right)^{1 / 3}-\frac{2}{f}\left(\frac{\phi}{\phi_{0}}\right)\right]
$$

Here, $\phi$ and $\phi_{0}$ are network volume fractions at equilibrium swelling and in a reference state, respectively. (2/f) (where $f$ is the functionality) 
is 0.5 for BIS, and almost 0 for clay in NC gels because of its high functionality. $V_{\mathrm{s}}$ is the molar volume of water. $\chi=\chi_{1}+\phi \chi_{2}$, where $\chi_{1}=(\Delta H-T \Delta S) / k_{\mathrm{B}} T, \quad \chi_{2}=0.518, \quad \Delta H=-12.462 \times 10^{-21} \mathrm{~J}$ and $\Delta S=$ $-4.717 \times 10^{-23} \mathrm{JK}^{-1}$. ${ }^{11}$ The calculated values of $v_{\mathrm{e}}$ for NC and OR gels are listed in Table 1. The effect of $C_{\text {clay }}$ on $v_{\mathrm{e}}$ in NC gels is analogous to that of $C_{\mathrm{BIS}}$ on $v_{\mathrm{e}}$ in OR gels; that is, $v_{\mathrm{e}}$ of $\mathrm{NC}$ gels increases with $C_{\text {clay }}$ In Table 1 , the $v_{\mathrm{e}}$ of soft $\mathrm{NCl}$ gel is about oneninth of that of the commonly used OR1 gel; even in the case of NC10 gel, which has excellent mechanical properties (initial modulus $(E)$ and tensile strength $(\sigma)), v_{\mathrm{e}}$ is only one-third of that of OR1 gel. The fact that values of $v_{\mathrm{e}}$ for NC gels are generally smaller than those of OR gels is consistent with the results obtained for the mechanical properties. Thus, it was concluded that, in NC gels, relatively small numbers of crosslinks are effectively formed on the surfaces of clay platelets.

Table 1 Degree of equilibrium swelling (DES) and effective network density $\left(v_{\mathrm{e}}\right)$ for NC and OR gels, calculated according to the Flory-Rehner theory, equation (2)

\begin{tabular}{lcccccccc}
\hline Hydrogel & \multirow{2}{*}{ NC1 } & \multirow{2}{*}{ NC5 } & NC10 & NC15 & NC20 & OR1 & OR5 \\
\hline DES $\left(W_{\mathrm{s}} / W_{\text {dry }}\right)$ & 50.94 & 28.94 & 22.57 & 17.61 & 12.94 & 16.93 & 8.55 \\
$v_{e}\left(\mathrm{moll}^{-1}\right)$ & 0.0048 & 0.0099 & 0.0127 & 0.0170 & 0.0256 & 0.0431 & 0.1582
\end{tabular}

\section{Mechanism of polymer/clay network formation}

The mechanism of forming the organic (polymer)/inorganic (clay) network structure of NC gels was elucidated on the basis of changes in viscosity, optical transparency, XRD and mechanical properties. ${ }^{31}$ Model structures relating to the process of forming NC gels are shown in Figure 6a. During the preparation of the initial reaction solutions, a specific solution structure was formed, in which the monomer (NIPA) largely prevents the gel formation of clay itself $(6 a(i))$, and initiator and accelerator are located near the clay surface through ionic interactions (6a(ii)). Thereafter, by increasing the solution temperature, free-radical polymerization was initiated by the redox system close to the surface of clay (6a(iii)). By this mechanism, we propose that 'clay-brush particles', composed of exfoliated clay platelets with numbers of polymer chains grafted to their surfaces, are formed in early stages of NC gel synthesis, as depicted in Figure 6a(iv)

The formation of clay-brush particles was confirmed by the time dependence of optical transmittance during in situ polymerization. A distinct reduction in transparency was observed in the very early stage of in situ polymerization (Figure 6b: N-NC2 gel), ${ }^{31}$ which may correspond to the formation of assemblies of clay-brush particles. Monomer conversion at the point of minimum transparency was $\sim 7 \%$, where the polymer/clay weight ratio was about 0.2:1. Transparency was reestablished on further polymerization. In contrast, no transparency changes were observed during the polymerization of OR a

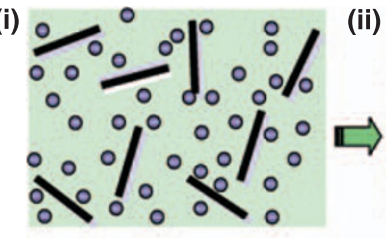

(ii)

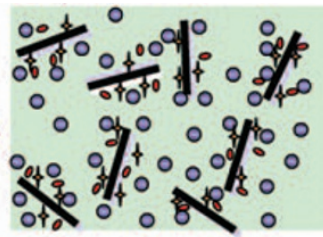

(iii)

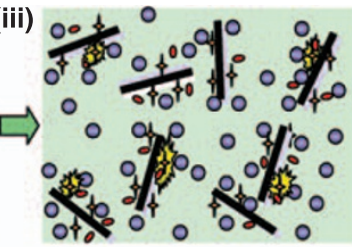

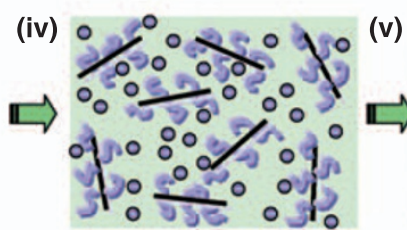
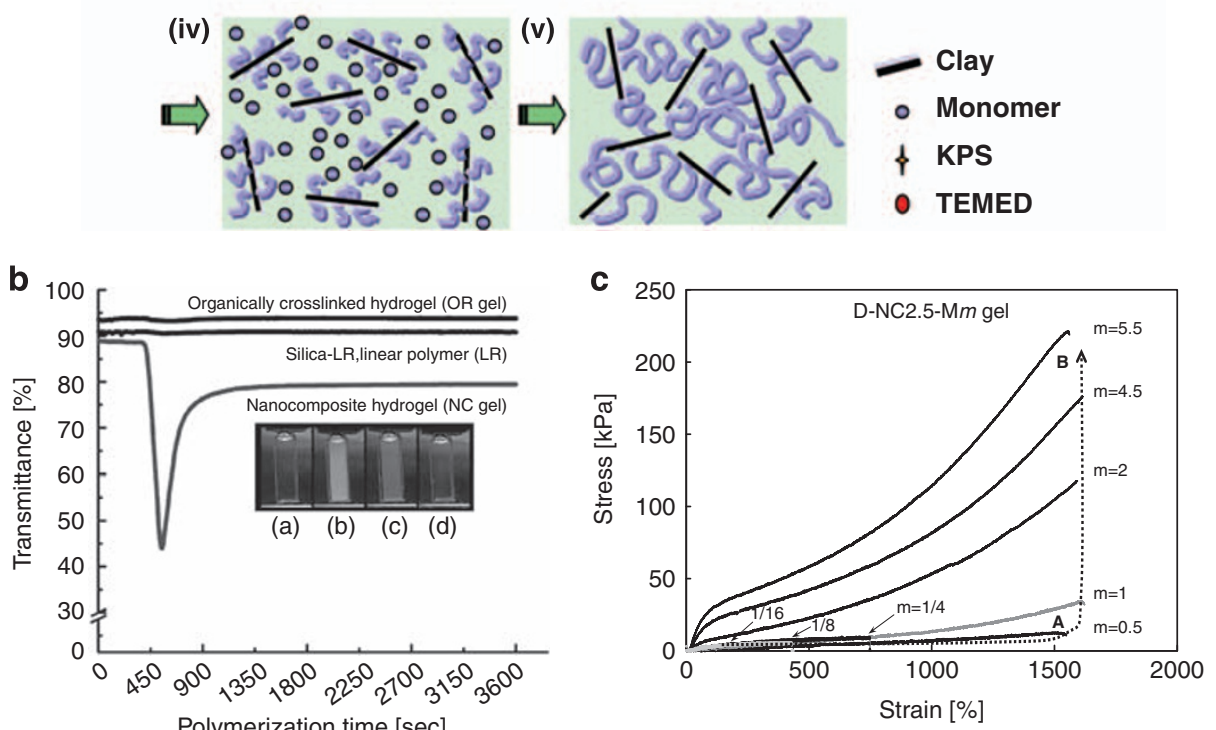

Figure 6 (a) Schematic representations of the model structures for the reaction solution and the mechanism of forming organic/inorganic network structure in a nanocomposite gel (NC gel). (i) Aqueous solution consisting of clay and N-isopropylacrylamide (NIPA). (ii) Reaction solution consisting of clay, NIPA, potassium persulfate (KPS) and $N, N, N^{\prime}, N^{\prime}$-tetramethylenediamine (TEMED). (iii) Radical formation near the clay surface in the reaction solution. (iv) Formation of clay-brush particles. (v) Formation of organic/inorganic networks. In the models, only a small number of monomer (polymer), KPS and TEMED are depicted for simplicity. (b) Changes of optical transparency during the polymerization of NC gel (N-NC2-M1 gel), OR gel (N-OR1-M1 gel), linear polymer (LR) and silica-containing LR. Photos (a)-(d) were obtained during the polymerization of NC gel. (c) Effects of polymer content $(m=1 / 16-5.5)$ on the stress-strain curves of D-NC2.5-Mm gel. ${ }^{31}$ 
gels or LR, or even in the case of PNIPA containing silica or titania particles (Figure 6b). Therefore, it was concluded that the newly observed transparency changes were a characteristic of NC gel synthesis, that is, the changes are related to the formation of clay-brush particles and the subsequent formation of an organic/inorganic network structure. This was also confirmed by XRD measurements. ${ }^{31}$

The proposed mechanism of forming the organic/inorganic network structure was supported by the fact that the stress-strain curves obtained for NC gels with different polymer contents exhibit characteristic two-step changes, as shown by the arrows in Figure $6 \mathrm{c}$ for D-NC2.5-Mm gels, ${ }^{31}$ which correspond to the formation of a primary network (arrow A: first step, $m \leqslant 0.5$ ) and a subsequent increase in the number of crosslinked polymer chains (arrow B: second step, $m \geqslant 1$ ). Furthermore, dynamic light scattering and SANS measurements showed that the gelation in NC gels is classified as an ergodic-nonergodic transition, as that in OR gels; the only difference is that huge clusters of NC microgels (corresponding to clay-brush particles) are formed before the gelation threshold in the case of the former. ${ }^{34}$

NC gels with excellent mechanical properties and structural homogeneity could not be prepared by other methods, such as those involving mixing of clay and polymer solutions, or by in situ polymerization in the presence of the other inorganic nanoparticles such as silica or titania. ${ }^{31}$ These results indicate that the formation of organic/inorganic network structures in NC gels is highly specific and is achieved only by in situ free-radical polymerization in the presence of clay. The specific role of clay, as distinct from silica, in the preparaton of NC gels is also confirmed from investigations of the nature of PNIPA after eliminating clay and silica from the gels. ${ }^{37}$

\section{BASIC PROPERTIES OF NC GELS}

The optical, mechanical and swelling/deswelling properties of NC gels are superior to those of conventional OR gels. Furthermore, these basic properties can be controlled as desired by altering the network composition or by modifying the network structures.

\section{Optical transparency}

The optical transparency of hydrogels generally reflects the spatial inhomogeneity in the networks. The main factors affecting the transparency of NC gels are the degree of dispersion of clay nanoparticles in aqueous media and clay/monomer or clay/polymer interactions. Translucent or opaque NC gels are obtained when clay is insufficently exfoliated in the reaction solution or when microscopic aggregations of clay and monomers or polymers are formed. The effect of the clay content $\left(C_{\text {clay }}=1-9\right)$ in N-NC gels, and that of the BIS content $\left(C_{\mathrm{BIS}}=1-9\right)$ in N-OR gels, on transparency measured at $1^{\circ} \mathrm{C}$ is shown in Figure $7 .^{20}$ Transparent OR gels generally become opaque with increasing $C_{\mathrm{BIS}}$, because of the inhomogeneous distribution of crosslinking points; the critical value above which the transmittance decreases changes with the type of polymer used (for example, $5 \mathrm{~mol} \%$ (N-OR) and $8 \mathrm{~mol} \%$ (D-OR)). In contrast, NC gels are generally transparent, regardless of $C_{\text {clay }}$ and $C_{\mathrm{p}}$ values or the type of polymer used.

\section{Mechanical properties}

For a long time, the tensile mechanical properties of hydrogels such as PNIPA hydrogels were not investigated, because hydrogels with chemically crosslinked networks (OR gels) cannot withstand mechanical stretching or bending as described in the former section. However, the creation of NC gels has made it possible to conduct all kinds of mechanical tests. The most striking characteristics of NC gels are their excellent mechanical properties such as high $\varepsilon_{\mathrm{b}}(>1000 \%)$, high

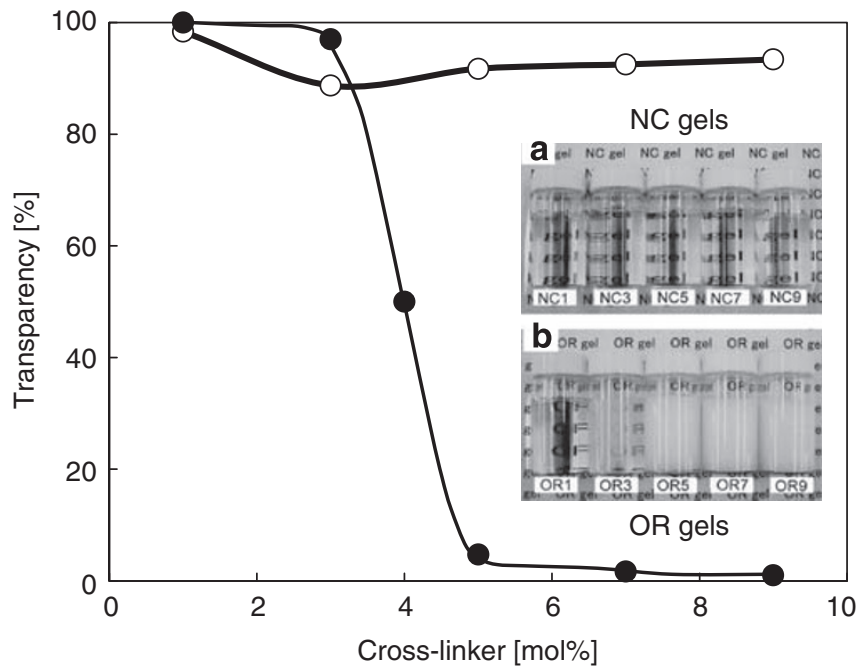

Figure 7 Changes in optical transparency of N-NC and N-OR gels produced by altering the crosslinker contents. Optical transmittance was measured at $1^{\circ} \mathrm{C}$ for hydrogels. ${ }^{20}$

tenacity (bending by more than $\left.360^{\circ}\right)$ and high $\sigma(>100 \mathrm{kPa})$; these properties were entirely unexpected and distinct from those of OR gels having the same composition, except for the type of crosslinker.

Figure $1 \mathrm{~b}$ shows the changes in the tensile stress-strain curves for NC gels with different $C_{\text {clay }}\left(C_{\text {clay }}=0.05-10\right) .{ }^{8}$ At low $C_{\text {clay }}(<1)$, $E$ increased slightly with $C_{\text {clay }}$ but $\varepsilon_{\mathrm{b}}$ decreased sharply to about $1000 \%$. However, when $C_{\text {clay }}$ was higher than that for NC1, $E$ and $\sigma$ increased greatly, as indicated by the dotted line, whereas values of $\varepsilon_{\mathrm{b}}$ were approximately constant $\left(\varepsilon_{\mathrm{b}} \approx 1000 \%\right)$. Thus, the effects of crosslinker, BIS and clay on tensile mechanical properties are totally different from each other. In NC gels, clay platelets have a specific role, functioning as effective multifunctional crosslinkers to form polymer/clay networks. The critical concentration $C_{\text {clay }}{ }^{\text {crit(1) }}{ }^{\text {, above }}$ which a well-defined organic/inorganic network is formed, is $\sim 1$, for the present system $\left(C_{\mathrm{NIPA}}=1 \mathrm{M}\right)$.

As the number of crosslinking units per unit volume in $\mathrm{NC}$ and $\mathrm{OR}$ gels is different (for example, 10 and 5400 units per $100 \mathrm{~nm}$ cube for NC3 and OR1, respectively), $D_{\text {ic }}$ in NC gels should be very large with a narrower distribution compared with those in OR gels. The reason for the high $\varepsilon_{\mathrm{b}}$ in NC gels, regardless of the high $C_{\text {clay }}$ is attributed to the high deformability of the long, flexible polymer chains in the unit structure of the polymer/clay networks (Figure $1 \mathrm{~b}(\mathrm{i})$ ). $E$ and $\sigma$ for NC gels also increased with increasing $C_{\mathrm{p}}$, as shown in Figure $6 c,{ }^{31}$ as the number of polymer chains linking neighboring clay platelets increases with $C_{\mathrm{p}}$.

Figure 8a shows tensile stress-strain curves for N-NC gels with different $C_{\text {clay }}$ (NC5-NC25) values, ${ }^{27}$ where both $E$ and $\sigma$ increase markedly with $C_{\text {clay }}$ as shown by the arrows. $\sigma$ exceeds $1000 \mathrm{kPa}$ in the $\mathrm{N}-\mathrm{NC} 25$ gel. The steep increase in $E$ at high $C_{\text {clay }}$ is probably due to the formation of rigid structures involving clay-clay interactions, similar to a house-of-cards structure ${ }^{42}$ (Figure $2 \mathrm{c}$ ) or nematic-like clay structure. ${ }^{40}$ In fact, the optical anisotropy of as-prepared NC gels is pronounced in NC gels with $C_{\text {clay }} \geqslant 10 .{ }^{27}$ Thus, the tensile mechanical properties can be controlled over a wide range by altering $C_{\text {clay }}$ and $C_{\mathrm{p}}$. It should be noted that the changes in mechanical properties of NC gels are unique, that is, totally different from those observed in normal polymeric materials. In the case of the latter, an increase in $E$ is generally accompanied by a decrease in $\varepsilon_{\mathrm{b}}$ because the increase is normally caused by the orientation of polymer chains or modification 


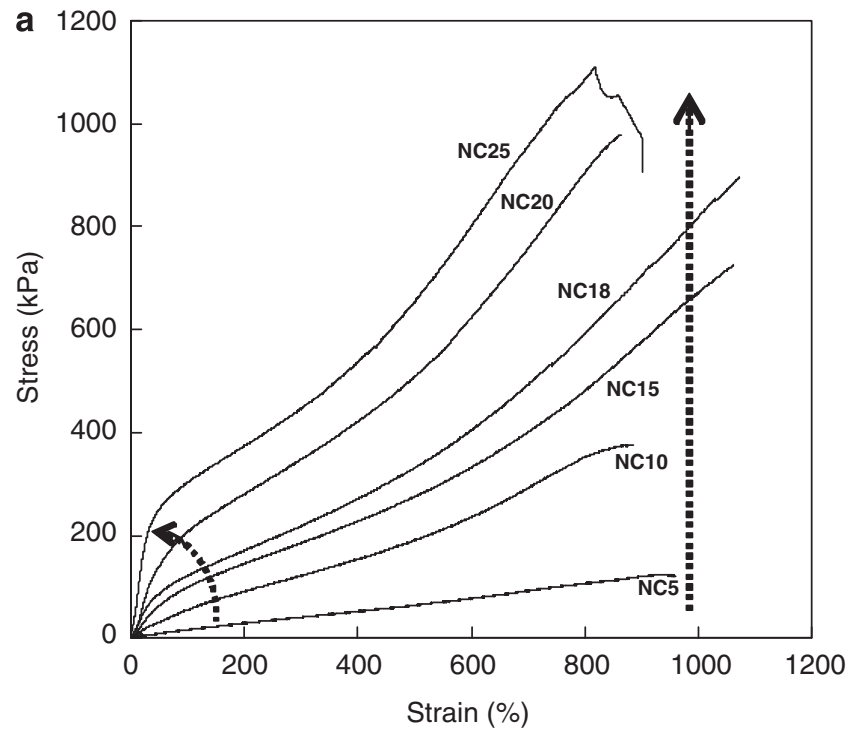

b

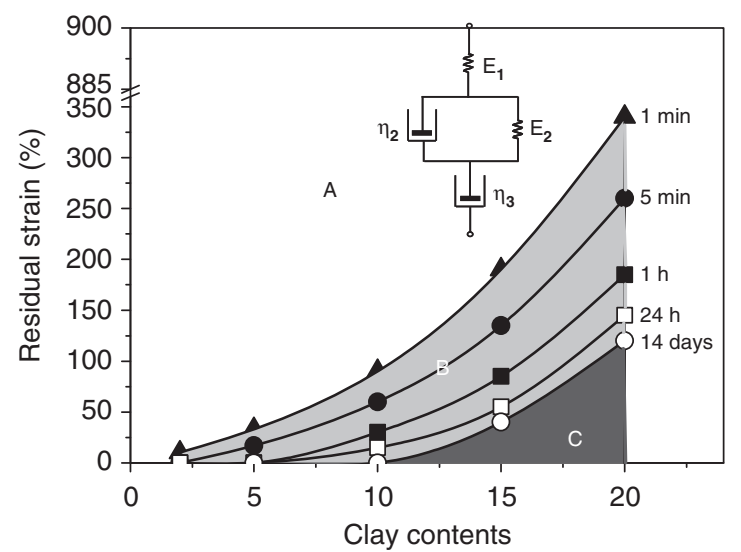

c

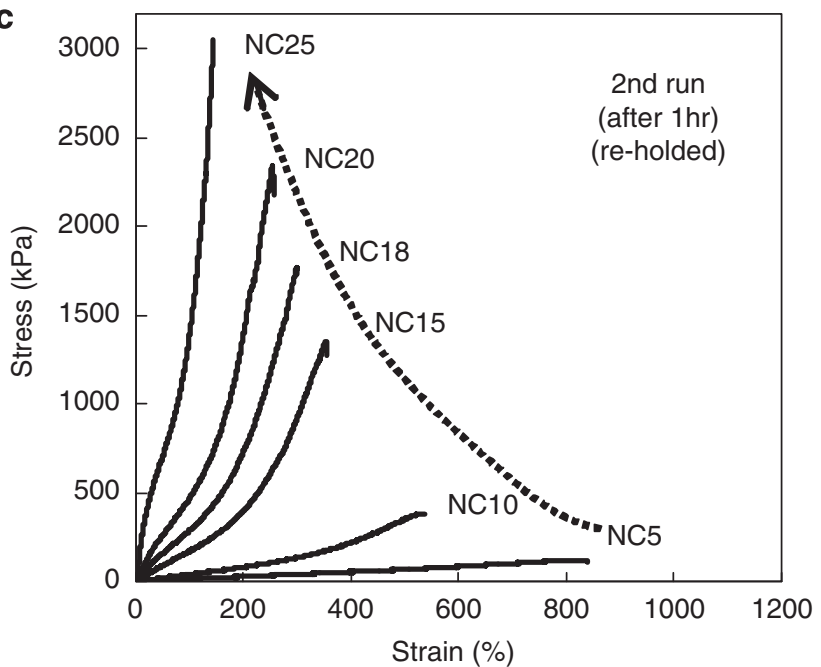

Figure 8 (a) Tensile stress-strain curves for N-NC gels with different $C_{\text {clay }}$ (NC5-NC25). All samples tested had the same ratio of water/polymer (10:1.13 w/w). (b) Time dependence of residual strain for NC gels with different $C_{\text {clay }}$ after release from stress from an initial elongation of $900 \%$ (or $800 \%$ for NC20 and NC25). A: quick recovery, B: time-dependent recovery, C: pseudopermanent strain. (Inset) Four-element mechanical model adopted for NC gels. (c) Tensile stress-strain curves for once-elongated NC gels with different $C_{\text {clay, }}$ which were prepared by elongation to $900 \%$ (or $800 \%$ for NC20 and NC25) and subsequently relaxed for 1 hour. ${ }^{27}$ of the polymer structure to a rigid one. In contrast, in NC gels, $E$ and $\sigma$ can increase without a large sacrifice of $\varepsilon_{\mathrm{b}}$ because of the polymer/ clay network structure. From the area under the tensile stress-strain curve, the fracture energies $\left(\xi_{\mathrm{f}}\right)$ for $\mathrm{NC}$ and $\mathrm{OR}$ gels and the dependences of $\xi_{\mathrm{f}}$ on the crosslinker content are determined. ${ }^{7,27} \xi_{\mathrm{f}}$ of NC gels increases in proportion to $C_{\text {clay }}$ whereas that of OR gels remains almost constant, regardless of $C_{\mathrm{BIS}}\left(\xi_{\mathrm{f}}=0.0014 \mathrm{~J}\right)$. The value of $\xi_{\mathrm{f}}$ for NC25 is nearly 3300 times that of OR gels. ${ }^{27}$ This is a very striking result, as $\mathrm{NC}$ and OR gels differ only in the crosslinker used.

NC gels showed characteristic recovery from high elongation. The time dependence of the residual strain after an elongation of $900 \%$ followed by immediate stress release is shown in Figure 8b: region A, instant recovery (within $1 \mathrm{~min}$ ); region $\mathrm{B}$, time-dependent residual strain; and region $\mathrm{C}$, pseudo-permanent strain (remains for more than 2 weeks). For N-NC gels with a low $C_{\text {clay }}$ ( $<\mathrm{NC10}$ ), 90-99\% instantaneous recovery was observed; on the other hand, for NC gels with high $C_{\text {clay }}(\mathrm{NC10}-20)$, the permanent strain $(C)$ gradually increased with $C_{\text {clay }}$ which is attributed to the irreversible orientation of the clay platelets. These elongation and recovery patterns of NC gels can be explained using a typical four-element mechanical model (inset of Figure 8b).

The stress-strain curves of N-NC gels in the second cycle were different from that observed in the first cycle (Figure 8c). ${ }^{27}$ For NC gels with higher $C_{\text {clay, }}$ remarkable increases in $E$ and $\sigma$ and a significant decrease in $\varepsilon_{\mathrm{b}}$ were observed in the second cycle. The effect of $C_{\text {clay }}$ on the $\sigma$ and $E$ of N-NC gels in the first and second cycles is summarized in Figure 9. ${ }^{27}$ The critical value of $C_{\text {clay }}$ crit(2) above which the mechanical properties in the second cycle become very different from those in the first cycle was found to be $\sim 10$. The change in the relative magnitudes of $E_{10-50}$ and $E_{100-200}$ was attributed to the rigidity of the as-prepared gels and the permanent orientation of the clay platelets (and PNIPA chains attached thereto) after the first elongation.

The tensile mechanical properties of NC gels could also be varied by changing the type of clay used. ${ }^{43}$ The modification of clay (for example, by fluorine substitution and/or by the addition of a dispersant) had a marked effect on the mechanical properties, where $E$ and $\sigma$ decreased but $\varepsilon_{\mathrm{b}}$ increased (Figure 10). Similar tendencies were also observed in the NC gels prepared using clay with a large particle size (montmorillonite: $>300 \mathrm{~nm}$ ). Further, the tensile mechanical properties can be modified by drying treatment, which is attributed to the additional crosslinks formed by irreversible rearrangement of the polymer/clay network structure in the concentrated state. ${ }^{44}$

NC gels can withstand high levels of deformation in all modes, including compression, torsion, tearing and bending, in addition to elongation. For example, NC gels can be tied into a knot without being damaged, and the knotted NC gel can be stretched without breaking at the knot (Figure $1 \mathrm{~b}(\mathrm{ii})){ }^{20}$ In compression tests, NC gels generally withstand a compression of up to $90 \%$ (Figure $1 \mathrm{~b}(\mathrm{iii})$ ). ${ }^{7,27}$ The compression modulus and strength of N-NC gels increase almost proportionally with $C_{\text {clay }}$. The strength reaches $5 \mathrm{MPa}$ at $80 \%$ strain for N-NC20 gels. $^{27}$

Furthermore, the ultimate tensile properties of rubbery $\mathrm{NC}$ gels changed considerably when the water content $\left(R_{\mathrm{H}_{2} \mathrm{O}}=W_{\mathrm{H}_{2} \mathrm{O}} / W_{\text {dry }}\right)$ was varied over a wide range (Figure 11). ${ }^{36}$ At a high $R_{\mathrm{H}_{2} \mathrm{O}}$, where PNIPA chains are fully hydrated, N-NC4 gels retain their rubbery tensile properties. However, when $R_{\mathrm{H}_{2} \mathrm{O}}$ is decreased, these gels undergo plastic-like deformation. Consequently, for a series of N-NC4 gels with different $R_{\mathrm{H}_{2} \mathrm{O}}$, a peculiar line (or 'failure envelope') was obtained by connecting the rupture points in the stress-strain 

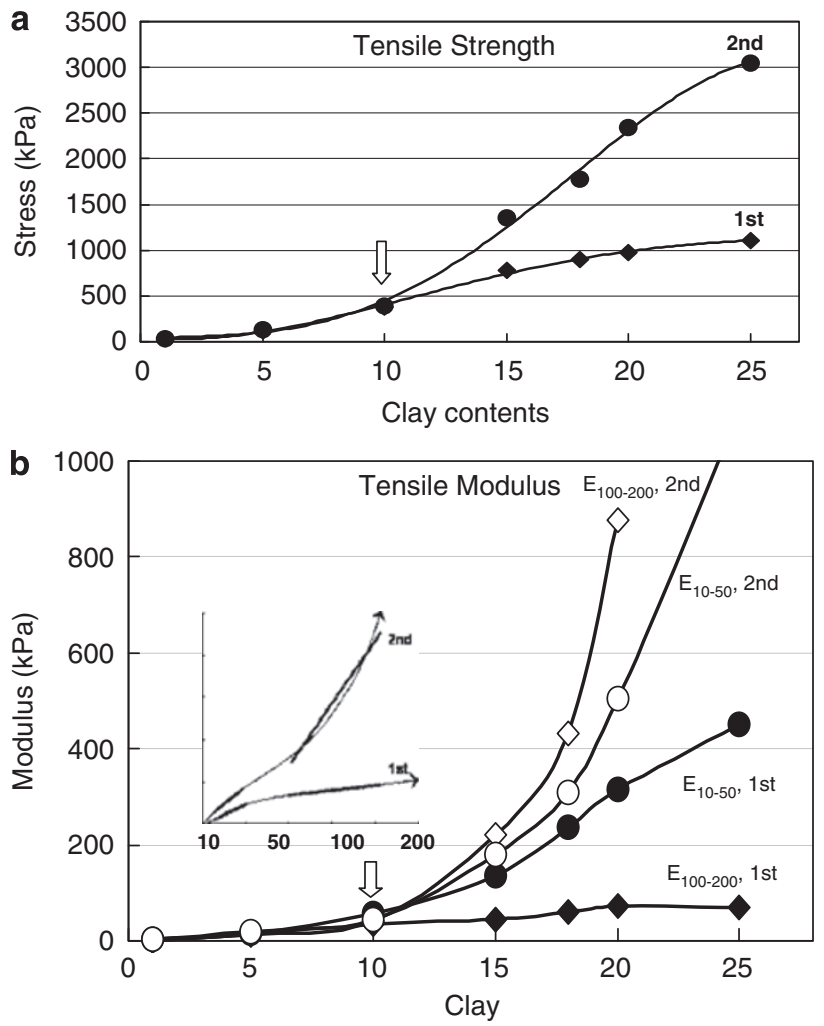

Figure 9 Changes in (a) tensile strength and (b) tensile modulus for two series of NC gels. As-prepared nanocomposite gels (NC gels) (first cycle: rhombus) and once-elongated NC gels (second cycle: circle). Two tensile moduli, $\mathrm{E}_{10-50}$ and $\mathrm{E}_{100-200}$, were calculated from the slopes in the ranges $10-50 \%$ and $100-200 \%$ elongation, respectively. The arrow indicates the critical clay concentration $\left(\mathcal{C}_{\text {clay }}{ }^{\text {crit(2) }}\right.$ ), above which the mechanical properties of the second cycle are largely changed. ${ }^{27}$

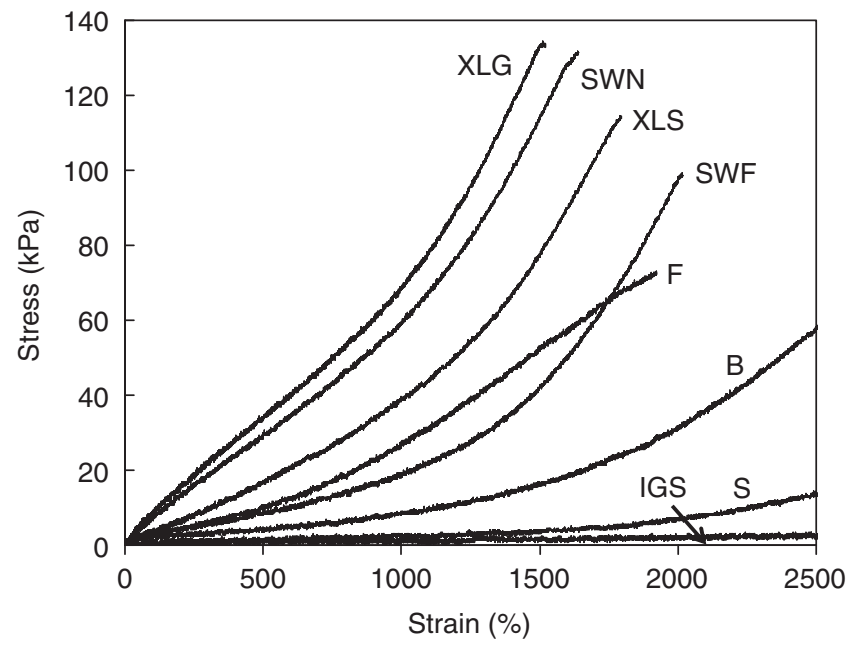

Figure 10 Stress-strain curves of D-NC gels prepared by using various kinds of clay: hectorite (XLG, SWN), fluorinated hectorite (SWF, B), hectorite modified with a dispersing agent (XLS), montmorillonite $(F), B+$ dispersing agent (S) and sepiolite (IGS). ${ }^{43}$

curves (Figure 11). Here, a counter-clockwise movement of the rupture point was observed on increasing the strain rate or decreasing $R_{\mathrm{H}_{2} \mathrm{O}}$. Thus, a decrease in $R_{\mathrm{H}_{2} \mathrm{O}}$ in NC gels produces an effect similar to that produced by a decrease in temperature in

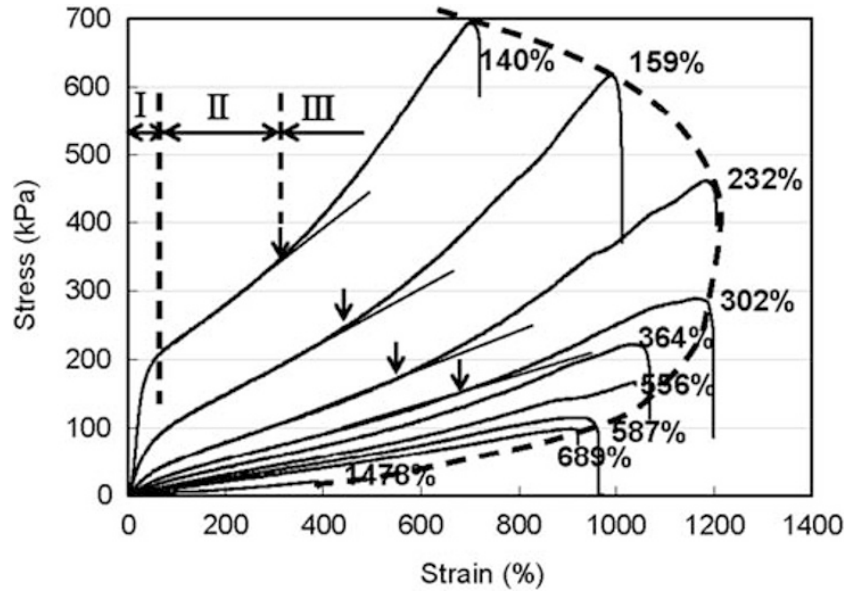

Figure $11 \mathrm{~A}$ series of stress-strain curves for N-NC4 gels with different $R_{\mathrm{H}_{2} \mathrm{O}}$ (wt\%). The numerical values are $R_{\mathrm{H}_{2} \mathrm{O}} \cdot R_{\mathrm{H}_{2} \mathrm{O}}$ for the as-prepared NC4 gel is $689 \mathrm{wt} \%$. A dashed line illustrates the failure envelope obtained by connecting the rupture points. The arrows represent the threshold strain points $\left(\varepsilon_{t}\right)$ above which the strain-hardening occurs. ${ }^{36}$

an amorphous elastomer (for example, styrene-butadiene rubber $(\mathrm{SBR})),{ }^{45}$ although the mechanisms are different in the two cases, namely entropic elasticity in the elastomer and plasticization in the NC gel.

While modifying the network structure by simultaneously using two crosslinkers, that is, inorganic clay and very small amounts of organic BIS, it was found that in co-crosslinked PNIPA hydrogels (NC-OR gels) the controllable region in the correlation between the compression strength and the modulus was expanded, as shown in Figure 12. ${ }^{29}$ This change was attributed to the formation of a microcomplex structure with relatively high chemical crosslink densities in proximiy to clay surface. Here, the combination of high strength and low modulus, which is often required for biomaterials but usually difficult to achieve in materials design, could be obtained.

\section{Stimuli sensitivities}

Thermosensitivity and its control in PNIPA hydrogels have attracted much attention because of their many potential applications. ${ }^{15-19}$ However, N-OR gels, consisting of chemically crosslinked networks, used so far had several important limitations, such as low volume change and slow deswelling rate, as well as poor mechanical properties. Among these disadvantages, slow rates of deswelling have been studied most extensively. As a result, fast deswelling has been achieved by introducing porosities, ${ }^{46}$ structural inhomogeneities ${ }^{47}$ or tailored graft structures ${ }^{48}$ into OR gels. However, in most cases, other properties such as mechanical properties, swelling ratio and optical transparency were not improved and were often made worse.

All these limitations are overcome by N-NC gels, which show large volume changes and high deswelling rates coupled with excellent mechanical properties. ${ }^{6,20} \mathrm{~N}-\mathrm{NC}$ gels, particularly those with low $C_{\text {clay, }}$ show outstanding stimulus sensitivity because the PNIPA chains in the network adopt flexible, random conformations, as described in the preceding sections. The deswelling kinetics for N-NC gels in water at $40^{\circ} \mathrm{C}$ (> LCST) and the effect of crosslinker content are shown in Figure 13a, and those for N-OR gels are shown for comparison in Figure $13 \mathrm{~b} .{ }^{20}$ As deswelling rates are inversely proportional to the square of typical gel dimensions and the initial samples used have large volumes $\left(712 \mathrm{~mm}^{3}\right)$, transparent $\mathrm{N}-\mathrm{OR}$ gels with low $C_{\mathrm{BIS}}$, such as $\mathrm{N}-\mathrm{OR} 1$ and $\mathrm{N}-\mathrm{OR} 2$, which are most commonly used as PNIPA 
hydrogels, undergo low degrees of deswelling; that is, the time taken to shrink is more than a month. In contrast, the N-NC1 gel exhibited the rapid response, and shrinking was almost complete within $10 \mathrm{~min}$. This is because, in the structurally homogeneous N-OR1 gel, the gel initially forms a hydrophobic, collapsed polymer skin on the macroscopic gel surface by eliminating near-surface water, through which the subsequent water permeation from the gel interior is very limited. In contrast, in NC gels with low $C_{\text {clay }}$ shrinking proceeds rapidly as water

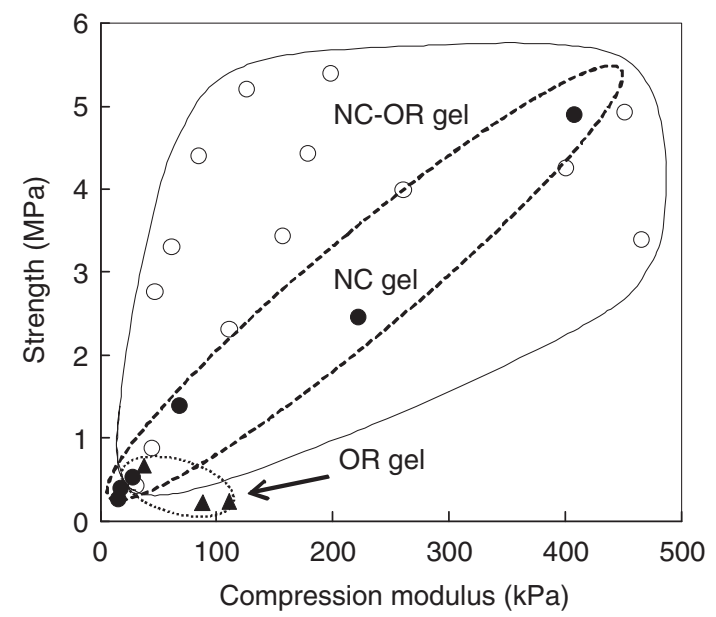

Figure 12 Correlation of compression strength and modulus, which is controllable in $\mathrm{NC} n, \mathrm{ORm}$ and $\mathrm{NC} n-\mathrm{OR} m$ gels by altering $n=0-10$ and $m=0-5$. The modulus-strength relations can be controlled within each rounded region. Here, the strength for NC and NC-OR gels is at $80 \%$ compression (without fracture), whereas the strength for OR gels is the maximum at break. ${ }^{29}$ is squeezed from the gel interiors through large numbers of water channels formed by micro-phase separation of the flexible PNIPA chains, including grafts. This concept is consistent with the fact that shrinking occurs rapidly when additional grafts are intentionally introduced into the chemically crosslinked networks. ${ }^{48}$

Another interesting point of note is that N-NC and N-OR gels exhibit entirely opposite tendencies with respect to the effects of crosslinker content $\left(C_{\text {clay }}\right.$ and $\left.C_{\mathrm{BIS}}\right)$ on the deswelling rate (Figures 13a and b). This is because, in NC gels, the mobility of PNIPA chains is gradually restricted as $C_{\text {clay }}$ increases, whereas the homogeneous network structure is retained. Therefore, the deswelling rate gradually decreases as $C_{\text {clay }}$ increases; this relationship differs from that stated in the Tanaka-Filmore theory. ${ }^{49}$ In contrast, in N-OR gels, deswelling rates increase with $C_{\mathrm{BIS}}$. This is due to the water changes formed in optically turbid (structually inhomogeneous) N-OR gels with high $C_{\mathrm{BIS}}$ as a result of heterogeneous distribution of crosslinking points. The different characteristics of N-NC and N-OR gels during swelling/ deswelling behaviors are clearly shown by experiments conducted using alternating temperature changes (Figure 13c). ${ }^{20}$ Here, although increases in $C_{\text {clay }}$ in N-NC gels resulted in decreased volume changes, increases in $C_{\mathrm{BIS}}$ in N-OR gels caused the volume change to increase.

With further increases in $\mathrm{C}_{\text {clay }}$ the degree of thermosensitive deswelling decreased markedly. ${ }^{7,40}$ As shown in Figure 14a, no volume contraction was observed for $\mathrm{N}-\mathrm{NC}$ gels with $C_{\text {clay }}>12$. Instead, NC gels underwent swelling, even at $50^{\circ} \mathrm{C}$ (>LCST), when PNIPA behaved as a hydrophilic polymer with no thermosensitive transition. ${ }^{40}$ Thus, volume changes on swelling or deswelling N-NC gels are controllable through large variations in $C_{\text {clay }}$. Analysis of the number of clay platelets per unit gel volume $\left(n_{\text {clay }}\right)$ in as-prepared NC gels (filled circles) and in gels stored at $50^{\circ} \mathrm{C}$ (open circles) showed that $n_{\text {clay }}$ at $50^{\circ} \mathrm{C}$ was almost the same ( $\approx 42$ per $10^{6} \mathrm{~nm}^{3}$ of gel) for all $\mathrm{NC}$
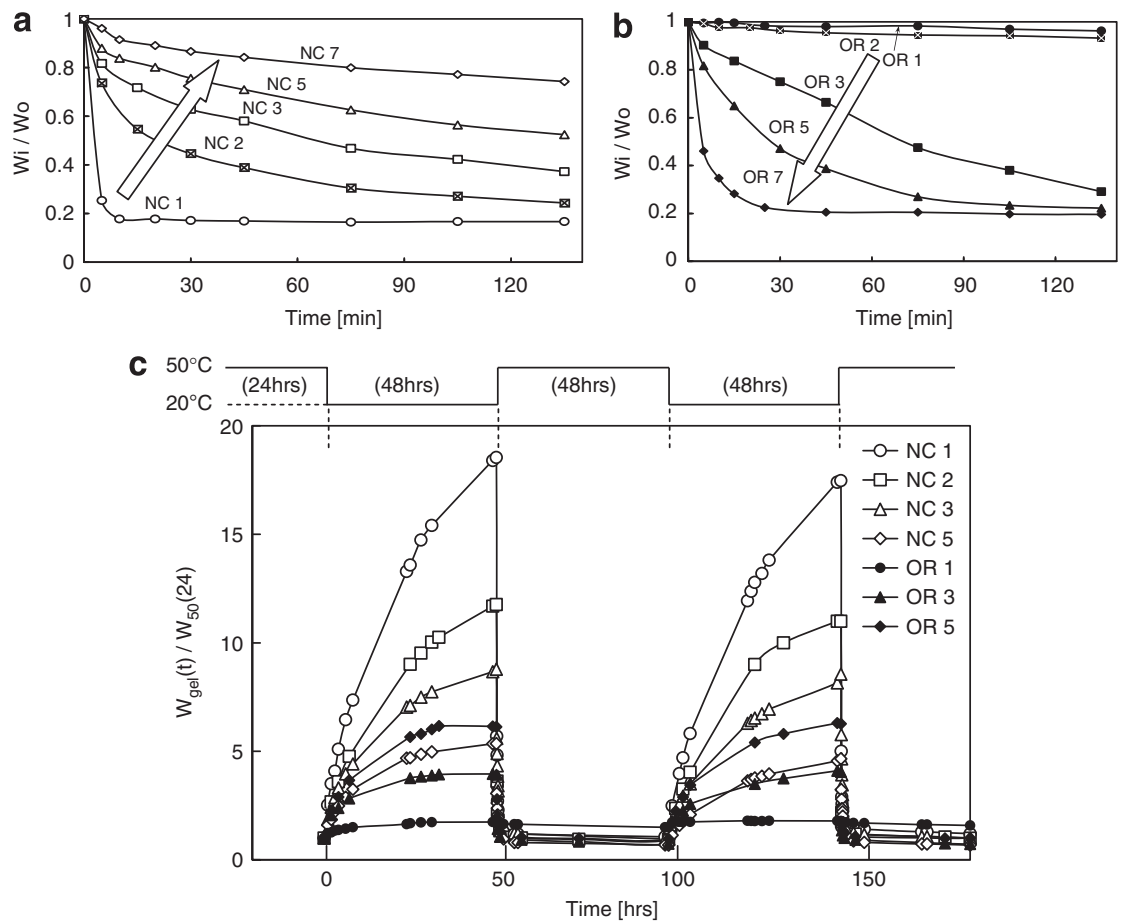

Figure 13 Deswelling kinetics (time dependences of weight) at $40^{\circ} \mathrm{C}$ for (a) $\mathrm{NC}$ gels and (b) OR gels with different crosslinker (clay and $N, N^{\prime}$ methylenebis(acrylamide) (BIS)) contents. All original gels had the same water/polymer ratio $(=10 / 1(\mathrm{w} / \mathrm{w}))$ and sample size $(5.5 \mathrm{~mm} \varphi \times 30 \mathrm{~mm}$ in length). (c) Swelling and deswelling behaviors by alternating temperature changes for nanocomposite gels (NC gels) and OR gels with different crosslinker contents. The time dependences of swelling ratio were measured as $W_{\text {gel }}(\mathrm{t}) / W_{50} .{ }^{24}$ Here, $W_{50}{ }^{24}$ is the gel weight after the first $24 \mathrm{~h}$ retention at $50^{\circ} \mathrm{C} .{ }^{20}$ 

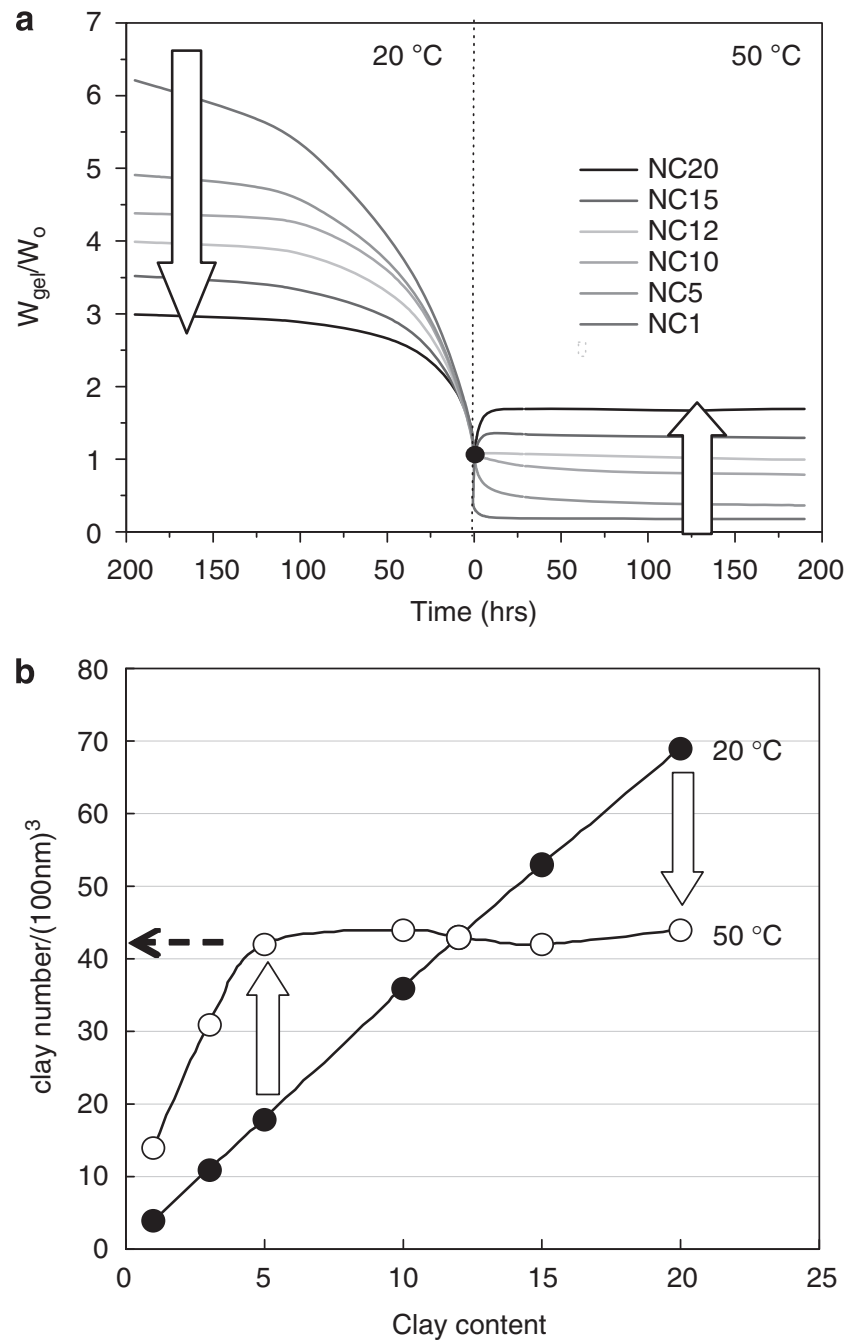

Figure 14 (a) Swelling/deswelling behaviors of nanocomposite gels (NC gels) with different $C_{\text {clay, }}$ measured in water at 20 and $50^{\circ} \mathrm{C}$. Changes in weight ratio $\left(W_{\text {gel }} / W_{0}\right)$ with elapsed time are plotted. $W_{0}$ is the initial weight of the as-prepared gel (initial size: $5.5 \mathrm{~mm} \phi \times 30 \mathrm{~mm}$ in length). (b) $C_{\text {clay }}$ dependencies of the number of clay platelets per unit of gel volume $\left(10^{6}\right.$ $\mathrm{nm}^{3}$ ) calculated for as-prepared $\mathrm{NC}$ gels at $20^{\circ} \mathrm{C}$ and for corresponding shrunken $\mathrm{NC}$ gels at $50^{\circ} \mathrm{C} .40$

gels with $C_{\text {clay }} \geqslant 5$ (Figure $14 \mathrm{~b}$ ); this observation indicates that during volume contraction, $n_{\text {clay }}$ increases to an upper limit of 42 per $10^{6} \mathrm{~nm}^{3}$. In addition, NC gels with high clay loadings in the asprepared state, such as $\mathrm{NC} 15$ and NC20, tend to expand even at $50^{\circ} \mathrm{C}$, until they reach the same value of $n_{\text {clay }}(\approx 42) .{ }^{40}$

With regard to changes in transparency of N-NC gels, decreases in transparency at the LCST varied dramatically with $C_{\text {clay }}$ as shown in Figure $15 .^{7}$ Thereafter, losses in transparency gradually decreased as $\mathrm{C}_{\text {clay }}$ increased. Eventually, for $\mathrm{C}_{\text {clay }}$ greater than $C_{\text {clay }}{ }^{\text {crit }(o p t(1))}(=10)$, there was no loss in transparency and gels remained transparent regardless of the temperature (the inset of Figure 15). ${ }^{7}$ This indicates that, in NC gels with $C_{\text {clay }}$ greater than $C_{\text {clay }}$ crit(opt(1)), thermoresponsive dehydration of PNIPA chains, that is, the conformational change to a globular, hydrophobic form, is hindered in PNIPA chains attached to, or lying close to, hydrophilic clay surfaces. This is the first observation of non-thermosensitive PNIPA hydrogels, in which the coil-to-globule transition of PNIPA chains is completely restricted by

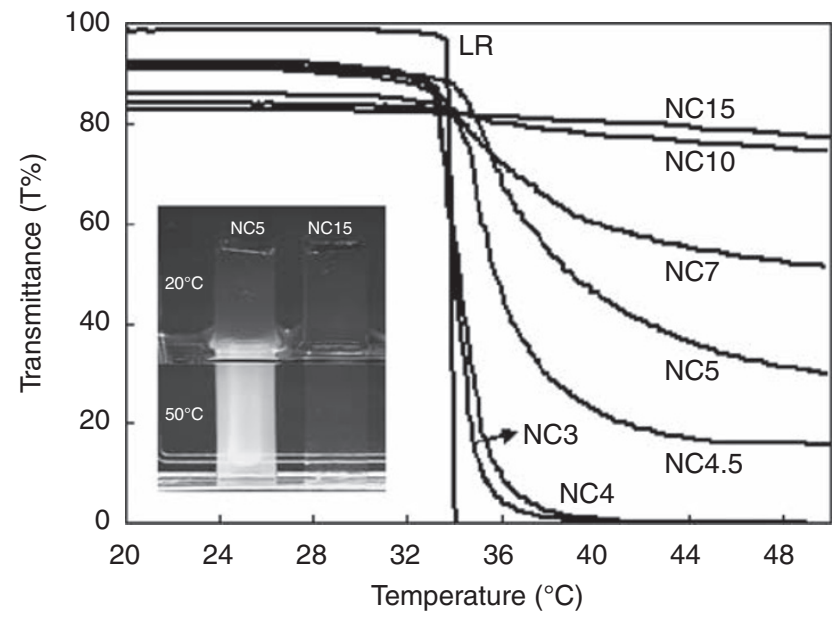

Figure 15 Temperature dependences of the optical transmittance for poly $(\mathrm{N}$ isopropylacrylamide) solution (LR) and nanocomposite gels (NC gels) with

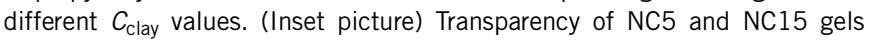
below (in air: upper) and above lower critical solution temperature (in water: lower). The transparency change observed for NC5 gel was not observed for NC15 gel. ${ }^{7}$

inorganic nanoparticles. The transition temperatures of NC gels, identified by half decreases in optical transmittance, increased with increasing $C_{\text {clay }}$ although the transmittance starts to decrease at a slightly lower temperature. ${ }^{40}$ Furthermore, the transition temperature decreased or increased markedly on adding an inorganic salt (for example, $\mathrm{NaCl}, \mathrm{CaCl}_{2}, \mathrm{AlCl}_{3}$ ) or a cationic surfactant (for example, hexadecyltrimethylammonium chloride) to the gel, respectively. ${ }^{50}$

To ensure that NC gels showed outstanding swelling/deswelling behavior in response to both temperature and $\mathrm{pH}$, while retaining their remarkable tensile mechanical properties, we prepared NC gels with a semi-interpenetrating organic/inorganic network structure by using linear poly(acrylic acid) (PAAc) ${ }^{51}$ The PAAc content $\left(C_{\mathrm{PAAc}}\right)$ required to achieve good mechanical properties, as well as good temperature and $\mathrm{pH}$ sensitivities, changed with $C_{\text {clay }}$ and the upper critical value of $C_{\text {PAAd }} / C_{\text {clay }}$ was $\sim 2.5-3$.

\section{NEW CHARACTERISTICS OF NC GELS}

\section{Optical anisotropy}

As polymeric hydrogels consist of amorphous polymer networks swollen with large amounts of water, the hydrogels are normally amorphous (optically isotropic). To date, optical anisotropy has only been studied in polymeric hydrogels containing mesogenic groups, ${ }^{52}$ or orientated by a flow-gelation process ${ }^{53}$ or an electric field. ${ }^{54}$ In contrast, NC gels exhibit optical anisotropy with increasing $\mathrm{C}_{\text {clay }}$ and uniaxial deformation, as they contain clay nanoparticles with an anisotropic, disk-like shape and are also capable of large deformations. For example, when $C_{\text {clay }}$ exceeds a critical value $\left(C_{\text {clay }}{ }^{\text {crit }}(\right.$ opt2 $\left.)=10\right), \mathrm{N}-\mathrm{NC}$ gels show optical anisotropy in the asprepared (unstretched) state, ${ }^{27}$ whereas $\mathrm{N}$-OR gels show no optical anisotropy at any value of $C_{\mathrm{BIS}}$. This $C_{\text {clay }}{ }^{\text {crit(opt2) }}$ is consistent with the critical value calculated for spontaneous clay aggregation (layer stacking) in NC gels. ${ }^{40}$ It should also be noted that $C_{\text {clay }}$ crit(opt2) is consistent with the other critical values of $C_{\text {clay }} C_{\text {clay }}{ }^{\operatorname{crit}(o p t(1)) 7,40}$ and $C_{\text {clay }}{ }^{\operatorname{crit}(2),}{ }^{27}$ described in the former sections.

The optical anisotropy of an NC gel changes in a unique manner on uniaxial deformation, regardless of its optical characteristics in the original (as-prepared) state; that is, when stretched uniaxially, all $\mathrm{N}-\mathrm{NC}$ gels show remarkable optical anisotropy, as can be seen in 

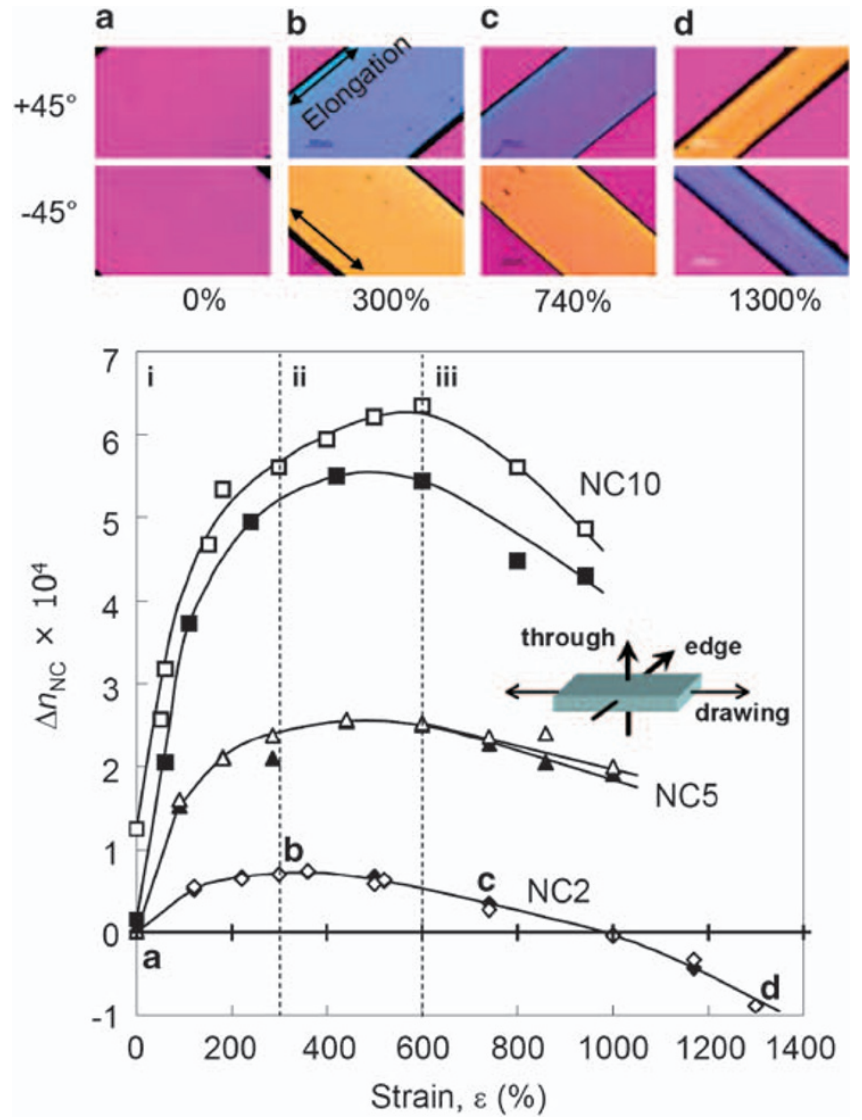

Figure 16 Birefringences, $\Delta n_{\mathrm{NC}}$, of nanocomposite gels (NC gels) with different $C_{\text {clay }}$ (NC2-NC10) values as functions of strain. Closed and open symbols were measured in through and edge directions (see inset Figure), respectively. Inserted photo images (a-d) show the polarized light micrographs for stretched NC2 gels (through directions) under crossed polarizers in conjunction with a $530 \mathrm{~nm}$ retardation plate, where $+45^{\circ}$ $\left(-45^{\circ}\right)$ orientation is parallel to the slow (fast) axis of the retardation plate. Each photo (a-d) corresponds to the same point on the $\Delta n_{\mathrm{NC}}$ strain curve of NC2 gel. ${ }^{55}$

Figure $16 .{ }^{55}$ Their birefringence $\left(\Delta n_{\mathrm{NC}}\right)$ strongly depends on $C_{\text {clay. }}$. An interesting point to be noted is that $\Delta n_{\mathrm{NC}}$ values show a distinct maximum at a strain of around $300-600 \%$ and a sign inversion on further elongation. Here, an NC2 gel stretched to its intersection point with the strain axis is optically isotropic (990\%: $\left.\Delta n_{\mathrm{NC}}=0\right)$, although it has definitely a highly oriented network structure. By evaluating the separate contributions of clay and PNIPA, assuming that $\Delta n_{\mathrm{NC}}=$ $\Delta n_{\text {PNIPA }}+\Delta n_{\text {clay }}$ we concluded that $\Delta n_{\text {clay }}$ increases rapidly in the early stages of elongation and saturates at $300-600 \%$ strain. On the other hand, $\Delta n_{\text {PNIPA }}$ changes monotonically increase in negative value when the NC gels are stretched. As a result, elongation of the chains brought about a decrease in the net birefringence and could nullify the contribution from the clay (positive $\Delta n_{\text {clay }}$ ), and eventually reverse the sign of $\Delta n_{\mathrm{NC}}$. Furthermore, values of $\Delta n_{\mathrm{NC}}$ for NC gels with high $C_{\text {clay }}\left(\geqslant C_{\text {clay }}{ }^{\text {crit }(\text { opt } 2)}\right)$ were different in different directions (Figure 16); this was attributed to the partial plane orientation of clay platelets. ${ }^{55}$ The orientations of clay and PNIPA during the stretching were confirmed by contrast-variation SANS measurements. ${ }^{56}$

\section{Sliding friction behaviors}

In general, as OR gels are readily damaged by sliding, sliding friction measurements have rarely been conducted at the surfaces of OR

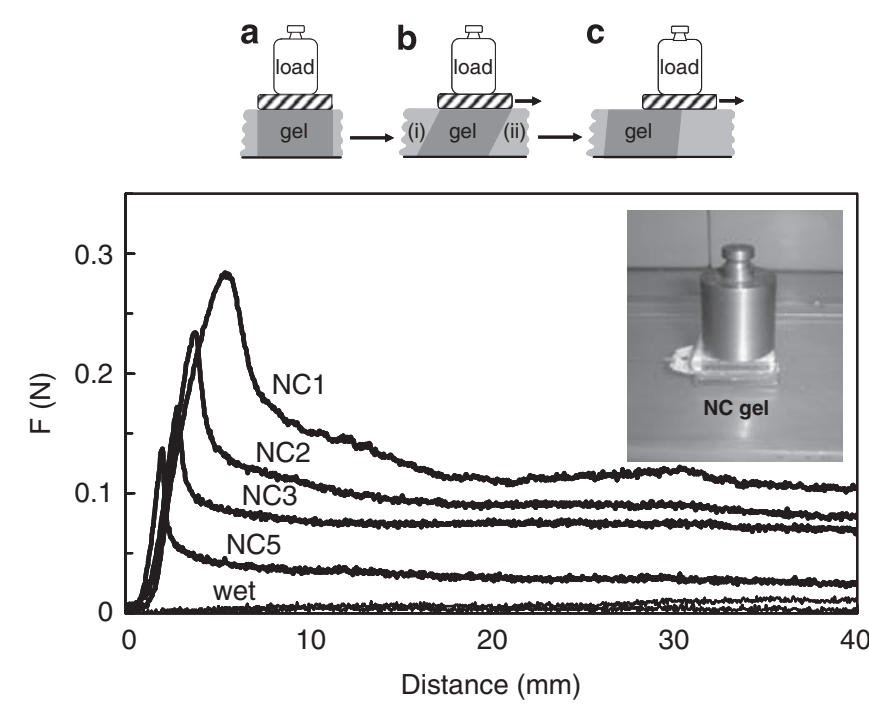

Figure 17 Effects of clay content of nanocomposite gels (NC gels) on the sliding frictional behavior. The force profiles were measured in air and wet for N-NC gels with different clay contents (NC1-NC5). (Inset) Schematic illustration (a-c) for the model of sliding process on $\mathrm{NC}$ gel, and a picture of sliding friction measurement on NC gel. ${ }^{57}$

gel films, except when films were wet. In contrast, NC gels are mechanically tough, and hence sliding friction tests have been conducted at their surfaces under different environmental conditions and even under high loading. Sliding frictional forces at NC gel surfaces are sensitive to the gel composition, loading and surrounding environment (wet, in-air and temperature; Figure 17). ${ }^{57}$ In air, NC gels show a force profile with a maximum static frictional force (max-SFF) and a subsequent constant dynamic frictional force. In contrast, NC gels show very low frictional forces when wet. The change in sliding friction behavior with the surroundings is most prominent for NC gels with low $C_{\text {clay }}$. For example, max-SFF for N-NC1 gel decreases by a factor $>10^{2}$ when its environment is changed from air to wet. Further, under wet conditions, the dynamic frictional coefficient, $\mu_{\mathrm{d}}$, decreases with increasing load and becomes very small at high loads $\left(\mu_{\mathrm{d}}<0.01\right)$. Thus, the frictional chracteristcs of N-NC1 gels can alternate between sticky and slippery depending on their surroundings. The sliding frictional forces for N-NC gels in air also decrease when they are heated to $50^{\circ} \mathrm{C}$ (>LCST), because of the coil-to-globule transition of PNIPA chains. ${ }^{30}$ The results are used to identify the important role of dangling chains at the gel-air and gel-water interfaces.

\section{High water contact angles}

Surface wettability is one of the most important properties of all materials, as it reflects the real structure and chemical composition at the outermost surface. As readily expected from their compositions, polymeric hydrogels, which consist largely of water and a hydrophilic polymer network, are naturally hydrophilic, and their surfaces generally show very low contact angles for water $\left(\theta_{\mathrm{w}}\right)$. The relation between the surface wettability of PNIPA hydrogels (N-OR gels) or surface-grafted PNIPA and the hydrophilic (coil)-to-hydrophobic (globule) transition occurring at the LCST has been studied extensively. ${ }^{58-60}$ It has been reported that $\theta_{\mathrm{w}}$ at the N-OR gel surface is low (for example, $\sim 60^{\circ}$ ) below the LCST but relatively high (for example, $\sim 80^{\circ}$ ) above the LCST. In contrast, N-NC gel surfaces showed extraordinarily high hydrophobicity (high $\theta_{\mathrm{w}}$ ) below the LCST (Figure 18), ${ }^{61}$ although all individual components of the N-NC gels were hydrophilic under the test conditions. Values of $\theta_{\mathrm{w}}$ for N-NC gels 
b $\quad 127.7^{\circ}$
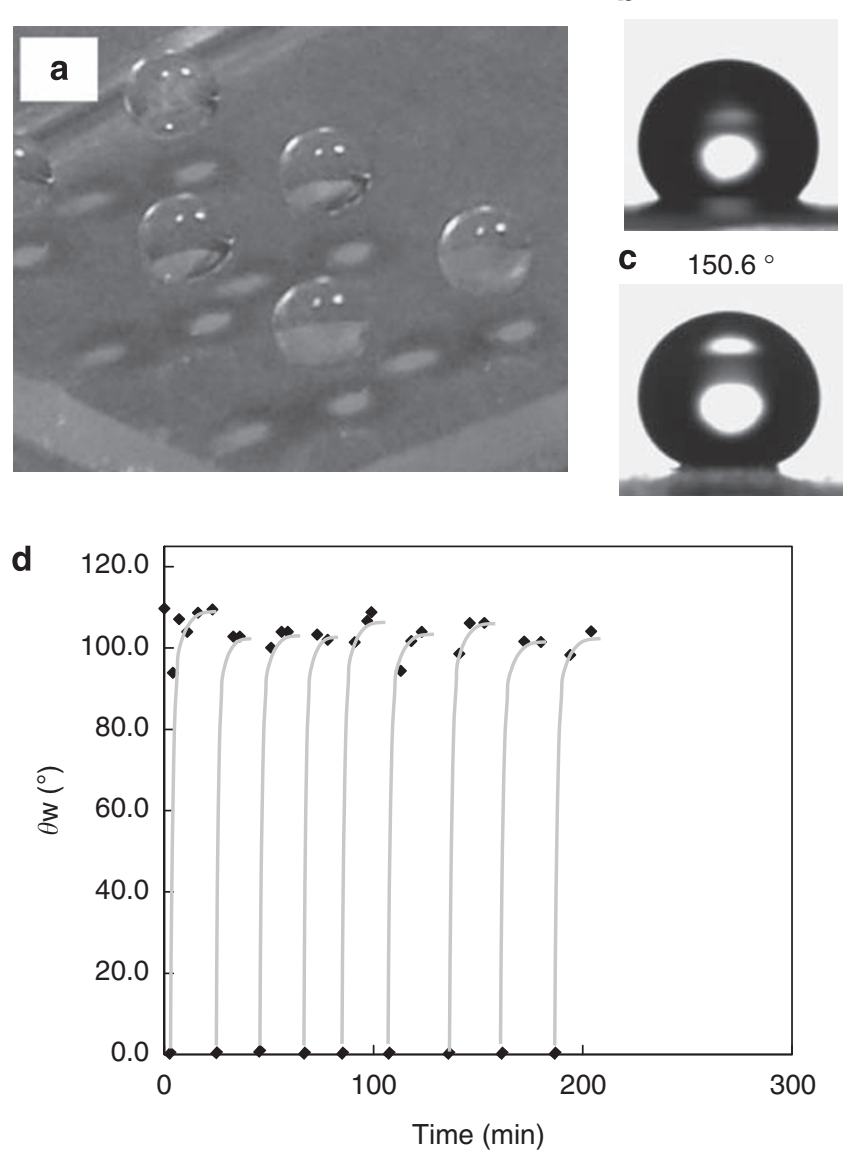

Figure 18 (a) Water droplets on N-NC6 gel film; (b, c) values of the water contact angles for sessile drops on the surfaces of N-NC6 gels with water contents of (b) $630 \mathrm{wt} \%$ and (c) $210 \mathrm{wt} \%$. (d) Changes in $\theta_{\mathrm{w}}$ by alternating between wet and dry conditions as defined in the text on the surfaces of N-NC5 gel (cross-section). ${ }^{61,62}$

were generally $>100^{\circ}$ for a broad range of $C_{\text {clay }}$ and $R_{\mathrm{H}_{2} \mathrm{O}}$ and reached a maximum of $151^{\circ}$ at a specific composition. ${ }^{61,62}$ These results were astonishing as values of $\theta_{\mathrm{w}}$ for N-NC gels were higher than those of polypropylene and poly(tetrafluoroethylene). The high hydrophobicity of N-NC gels was primarily attributed to the amphiphilicity of PNIPA and, more specifically, to the spontaneous alignment of $N$-isopropyl groups at the gel-air interface. ${ }^{61,62}$ The hydrophobicity was also enhanced by other factors such as the network structure and water content, whereas the effect of surface roughness was negligible. It was also found that the surfaces of N-NC gels underwent reversible hydrophobic-to-hydrophilic changes when the environment was changed from air to wet, and vice versa (Figure 18d). ${ }^{62}$

\section{Cell cultivation}

Culturing cells on a substrate is one of the most important and indispensable experimental procedures in medical, biological, pharmaceutical and tissue engineering researches. It was known that N-OR gels ${ }^{63}$ or PNIPA coatings of thickness $>30 \mathrm{~nm}^{64}$ cannot be used as substrates. However, it was found that cells can be cultured on the surfaces of normal or dried N-NC gels, regardless of gel thickness. ${ }^{65}$ To the best of our knowledge, this is the first report on the successful culturing of cells on a PNIPA hydrogel. Various types of cells, such as human hepatoma (HepG2) cells, normal human dermal fibroblasts and normal human umbilical vein endothelial cells, were cultured to be confluent on the surface of an N-NC6 gel. The development of the cell cultures showed little dependence on the water content or thicknesses of the N-NC gel sheets. In contrast, cell cultures did not develop on the surfaces of N-OR gels, regardless of the crosslinker content. Cell culture on the surfaces of D-NC and D-OR gels also failed, probably because of the hydrophilic nature of the gel surfaces. Hence, it was thought that cells adhered to and proliferated only on the surfaces of N-NC gels because of the combined effects of the hydrophobicity of the dehydrated PNIPA chains and the surface charges on the incorporated exfoliated clay. Furthermore, it was found that when the temperature was decreased to below the LCST $\left(10-20^{\circ} \mathrm{C}\right)$, the cell cultures detached from the surfaces of N-NC gels without trypsin treatment. ${ }^{65}$ Confluent layers of HepG2 and normal human dermal fibroblast cells could be spontaneously separated as sheets from the gel surface (Figure 19).

\section{Porous NCs with a layered morphology}

Novel, porous nanocomposites (porous-NCs) with characteristic layered morphologies were prepared by freeze-drying NC gels without the use of a porogen. ${ }^{66}$ The most typical morphology was a concentric three-layer morphology consisting of successively a fine-porous layer, a dense layer and a coarse-porous layer from the exterior to the interior (Figure 20). In the coarse layer, a regular assembly of polyhedral pores was formed spontaneously. A possible mechanism underlying the spontaneous formation of the unique three-layer morphology and other morphologies during the freeze-drying process was proposed.

\section{Reversible force generation}

Using temperature-sensitive N-NC gels, we discovered the generation of reversible retractive tensile forces in samples constrained to constant length in an aqueous environment in response to alternating temperature changes (Figure 21). ${ }^{67}$ This is the first observation of a retractive mechanical force generated as a result of conformational changes (coil-to-globule transition) in the PNIPA chains across the LCST. In contrast, no such force was generated in the case of D-NC and N-OR gels under the same experimental conditions.

\section{Complicated shapes and surface patterns}

In addition to various shapes, NC gel films with a wide range of thicknesses (thickness: $10^{-3}-10^{3} \mathrm{~mm}$ ) and sizes can be prepared. Further, NC gels with uneven surfaces, for example, surfaces with a regular array of pillars (Figure 3e) or bellows-like rods (Figure 3f), can be obtained. ${ }^{8}$ Here, bellows-like rods can be prepared by simply extracting the as-prepared NC gel from the mold without breaking or opening the template; this is because the protuberances on the surface are flexible and can be reversibly deformed during extraction. Further, by the direct replica molding, we successfully formed micrometer-scale surface patterns of NC gels (Figure 3g: 3D image obtained by laser-scanning confocal microscopy). ${ }^{68}$ The sizes of the patterns could be modified (enlarged or miniaturized) by subsequent swelling, deswelling and drying. OR gels with the aforementioned shapes and forms are difficult to prepare and handle because of their brittleness.

Photo-NC gels can also be prepared in various forms, including thin films and nanocoatings on a substrate, which exhibit additional characteristics such as pattern formation using photolithography, cell harvesting and fabrication of a microchannel flow system containing a thermosensitive valve made of photo-NC gels. ${ }^{30}$

\section{Other characteristics}

The other characteristics of NC gels that make them advanced and environmentally friendly materials are summarized as follows. (a) NC 

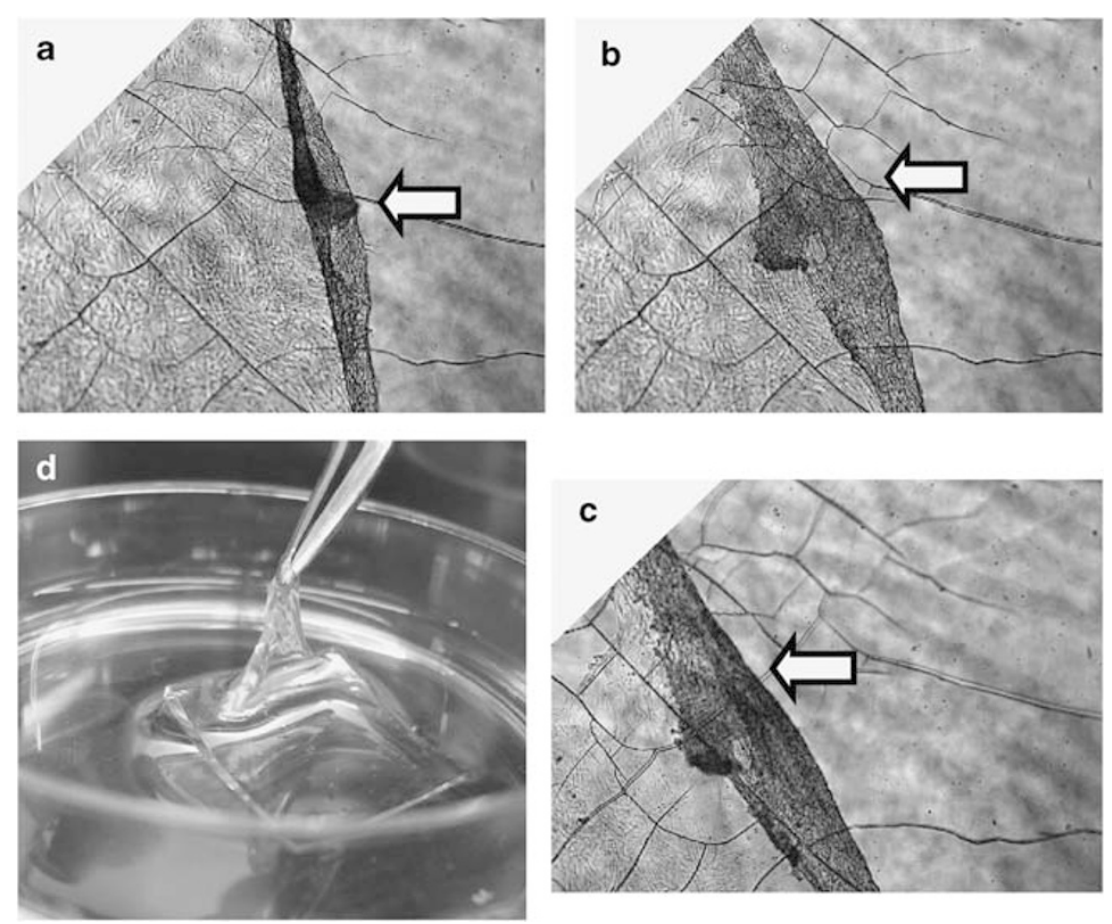

Figure 19 Cell sheet detachment of normal human dermal fibroblast (NHDF) by decreasing temperature to $10-20^{\circ} \mathrm{C}$. (a-c) Changes in cell sheet detachment from dried N-NC6 gels. (d) NHDF cell sheet. ${ }^{65}$

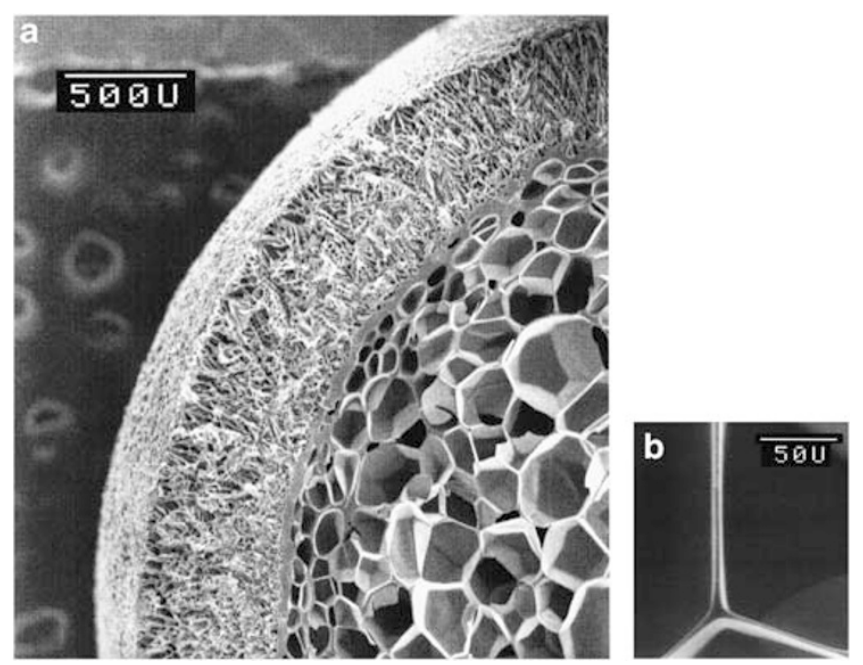

Figure 20 (a) Three-layer morphology observed by s.e.m. for a cross-section of porous-NC (freeze-dried NC gel in liquid nitrogen). Fine-porous, dense and coarse-porous layers were observed from the outer surface to the interior. (b) A junction between adjacent polyhedral pores in the coarseporous layer. The bar indicates 500 and $50 \mu \mathrm{m} .{ }^{66}$

gels exhibit capabilities of sewing, absorption (saline and excudate), sterilization (in an autoclave), safety (in vitro cytotoxicity tests), implantation and blood compatibility. ${ }^{69}$ (b) NC gels are virtually incombustible, even though they have no harmful halogen or phosphate moiety, and show excellent flame retardancy because their primary component is water. Dried NC gels are also expected to show fire retardancy, similar to polyamide/clay nanocomposites, ${ }^{70}$ because of their high clay content. (c) NC gels show fairly good heat conduction without additives, because of heat flow through their contained water. Further, NC gels have high heat capacity as water has the highest specific heat. (d) Transparent NC gels can be colored during synthesis or by posttreatment. (e) Lightweight NC gels, that is, porous NC gels, which are very soft (very low modulus) and have a density of $0.05-1.0 \mathrm{~g} \mathrm{~cm}^{-3}$, are prepared using a porogen or air (Figure 3i). (f) NC gels are ideal environmentally friendly materials ${ }^{9}$ as they are synthesized using water as the primary raw material (almost $90 \mathrm{wt} \%$ ).

\section{SOFT POLYMER NANOCOMPOSITES}

P-NCs with high clay contents

As described in the Introduction, the P-NCs developed so far as advanced composites are mainly obtained by the dispersion of small amounts of inorganic nanoparticles in a polymer matrix. ${ }^{2,4,5}$ There are a few reports of P-NCs with high inorganic content, such as nylon66/ silica (or clay) P-NCs and epoxy resin/silica P-NCs. ${ }^{71,72}$ However, these P-NCs still have certain limitations, particularly in terms of optical transparency, tractability and processability.

We have developed transparent, soft P-NCs with high contents of inorganic clay by extending the concept of NC gels and their synthesis to the field of solid P-NCs. These novel P-NCs (abbreviated as M-NCs), ${ }^{11,73}$ which consist of hydrophobic poly(2-methoxyethyl acrylate) (PMEA) and hydrophilic inorganic clay (hectorite), have superior optical and mechanical properties, despite their high $C_{\text {clay }}$ because of the unique organic/inorganic network structure composed of aggregated clay and PMEA. Here, PMEA is basically a hydrophobic polymer with a low $T_{\mathrm{g}}\left(-34^{\circ} \mathrm{C}\right),{ }^{74}$ and is considered to have promising applications in medical devices such as cardiopulmonary bypass, ${ }^{75-77}$ although in practical applications PMEA has only been used as an ingredient of copolymers or as a thin coating because of its mechanical weakness and intractability. Further, it was found that M-NCs show thermoresponsible cell adhesion/detachment useful for a living cell harvest system. ${ }^{12}$ 


\section{Synthesis of M-NCs}

A new type of soft P-NC (M-NC) was synthesized using a modified version of the preparative method for NC gels, that is, by in situ freeradical polymerization of water-soluble MEA in the presence of exfoliated clay and subsequent drying. Here, the essential point of the synthesis is that hydrophilic (monomer)-to-hydrophobic (polymer) transitions occur during in situ polymerization along with micro- and macro-phase separations. The initial transparent solution turned opaque (white) in the early stages of polymerization (within a few minutes) as a result of microscopic phase separation,

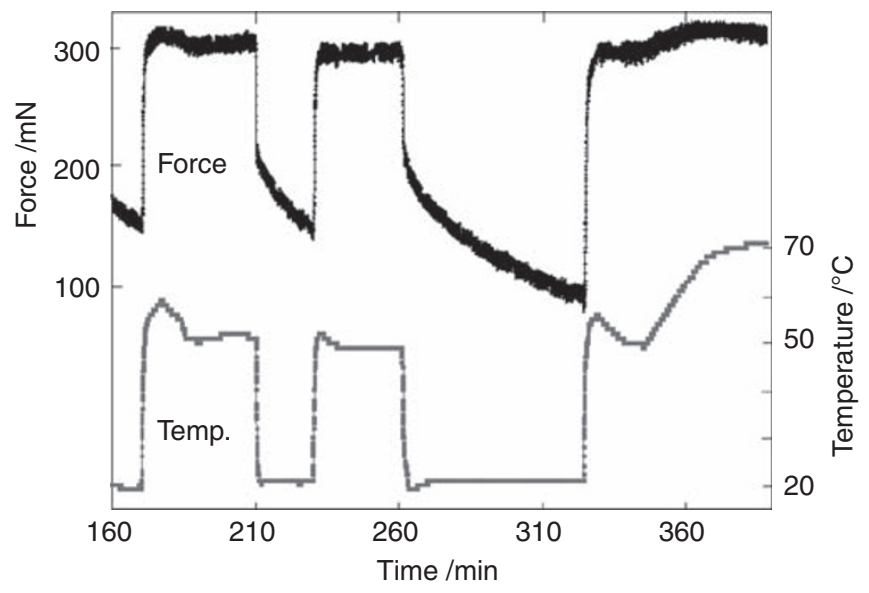

Figure 21 Force profiles for N-NC2-M2 gel by alternating temperature changes. 67 which was caused by exclusion of the unaltered hydrophilic clay from the hydrophobic PMEA chains. Subsequently, the system underwent macroscopic phase separation (after a few tens of minutes), which was accompanied by syneresis and volume contraction. Consequently, a uniform, white gel consisting of nanostructured PMEA/clay with $400 \mathrm{wt} \%$ water (relative to the solid component) was obtained. In the subsequent drying process, the white gel first shrank, releasing a large amount water, and finally turned into a transparent soft solid (M-NC). The resulting M-NCs were always uniform, colorless and transparent ( $>90 \%$ transmittance), regardless of $C_{\text {clay, }}$ as shown in Figure 22a (M-NC23). ${ }^{11}$ The water uptake on immersion in water was only $0.5-15 \mathrm{wt} \%$. This is totally different from NC gels, ${ }^{6,7,26}$ in which dried NC gels can revert to highly swollen hydrogels. In addition, M-NCs did not dissolve even in good solvents for PMEA, but could swell uniformly and extensively $\left(W_{\text {solvent }} / W_{\text {dry }}=2-25\right)$. These results strongly indicate that some kind of stable, 3D network is formed in M-NCs, although no organic crosslinking agent was used in their synthesis. The sample code for M-NCs is based on the clay content $\left(C_{\text {clay }}: n \mathrm{wt} \%\right)$, as $\mathrm{M}-\mathrm{NC} n$. $C_{\text {clay }}$ can be varied over a wide range (1-50 wt $\%$ or higher) by altering the composition of the reaction solution.

\section{Mechanical properties of M-NCs}

M-NCs have extraordinary mechanical properties, with two striking aspects: (1) an extremely large $\varepsilon_{\mathrm{b}}$, as high as $1000-3000 \%$, with good recovery on release; and (2) a well-defined yielding behavior in the early stages of elongation, as shown in Figure 23. ${ }^{11}$ The high extensibility of M-NCs is accompanied by a distinctive yielding behavior: a yield point $\left(\mathrm{I}_{\mathrm{b}}\right)$ at maximum stress, a necking region (II) at constant stress and a strain-hardening region (III) from the end
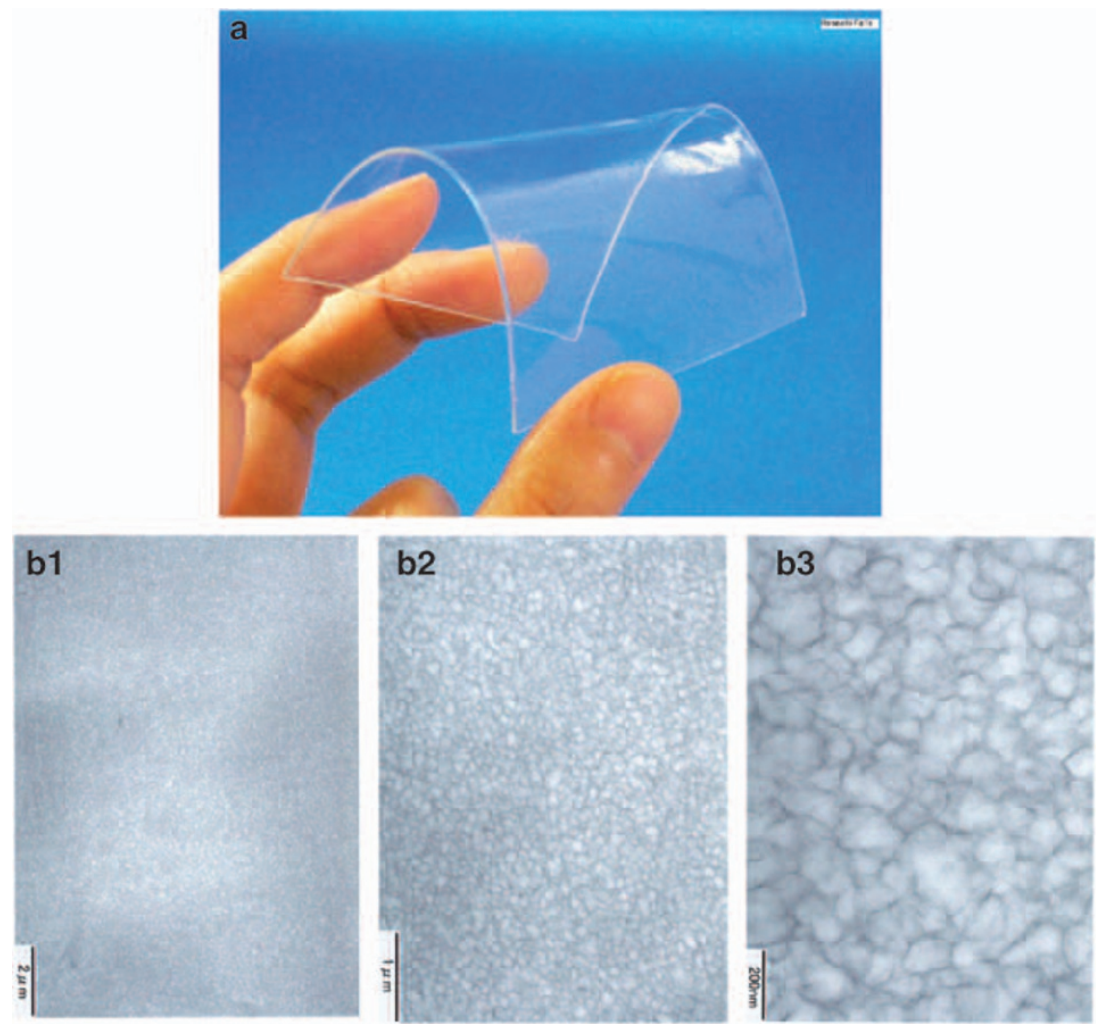

Figure 22 (a) Transparent and soft M-NC23 films that consist of 23 wt\% inorganic clay. (b) The transmission electron micrograph for M-NC11. (b-1), (b-2) and (b-3) are at different magnifications and scale bars indicate $2 \mu \mathrm{m}, 1 \mu \mathrm{m}$ and $200 \mathrm{~nm}$, respectively. ${ }^{11}$ 

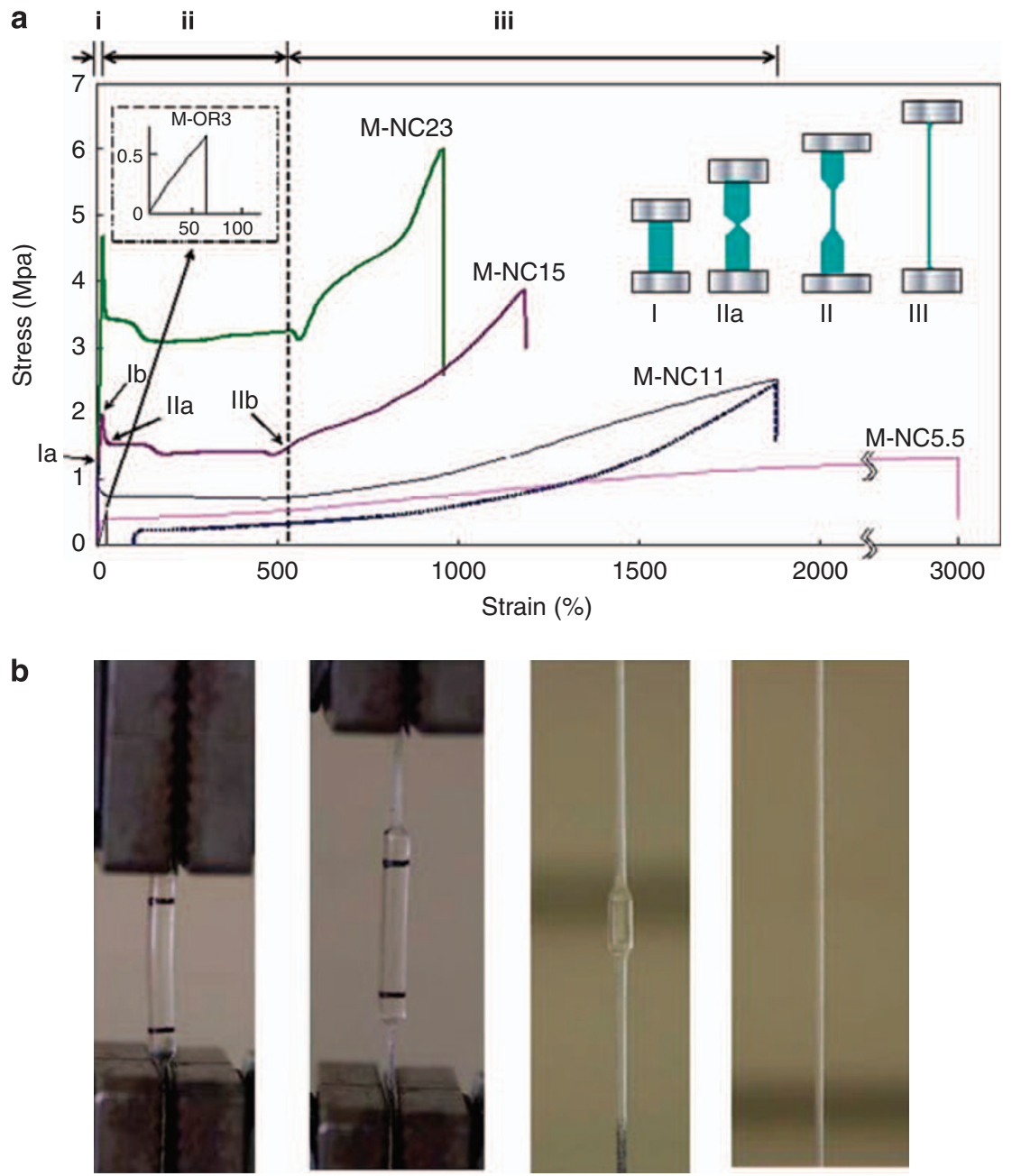

Figure 23 Tensile mechanical properties of M-NCs. (a) Stress-strain curves for M-NCs with different clay contents (M-NC5.5-23) and chemically crosslinked M-OR3. A stress-strain curve for the second cycle of M-NC11 after the first elongation up to $1800 \%$ is also shown as a dotted line. Schematic illustrations of typical yielding behaviors with well-defined necking points are depicted as inset. (b) Actual example of yielding behaviors observed for M-NC11 with two necking points that start at both ends in which a high stress concentration initially occurred at the grips. ${ }^{11}$

of the necking region $\left(\mathrm{II}_{\mathrm{b}}\right)$. This is evident from the stress-strain curve (Figure 23a) and direct observations (Figure 23b). This characteristic yielding behavior is observed for all M-NCs with different $C_{\text {clay, }}$ although it is more pronounced in M-NCs with $C_{\text {clay }}>10$. On the other hand, M-ORs with the same composition as M-NCs, except for the crosslinker used, were brittle and ruptured at very low elongation (M-OR3 in Figure 23a). The fracture energy of M-NC23 could be 200 times greater than that of M-OR3.

In M-NCs, more than $90 \%$ of the total elongation is recovered. Once elongated beyond point $\mathrm{II}_{\mathrm{b}}$, an M-NC exhibits simple stressstrain behavior; that is, it undergoes large deformation similar to the original (as-prepared) M-NC, although no well-defined necking phenomenon is observed (Figure 23a (dotted line) for M-NC11). Thus, the large deformation associated with necking becomes reversible on repeated cycling, but the necking phenomenon (that is, the formation of a neck) itself is irreversible. This is the first report on necking behavior in P-NCs. In extensive studies in polymeric materials such as crystalline polymers and high-impact polymer blends, the yielding (necking) behavior and associated large deformation were generally observed as a type of cold drawing, which was irreversible.

\section{Network structures in M-NCs}

To explain the origins of the dramatic changes in the mechanical properties of M-NCs, while retaining high transparency and low water absorption, we proposed the formation of nanostructured M-NCs on the basis of the analytical data, such as differential-scanning calorimetry ( $T_{\mathrm{g}}$ is the same as for free, uncrosslinked PMEA), XRD (evidence for stacking of clay-polymer-clay $(2.7 \mathrm{~nm})$ and clay-clay $(1.1 \mathrm{~nm})$ aggregates), Fourier-transform infrared spectroscopy (PMEA/clay interactions) and transmission electron microscopy. ${ }^{11}$ As the transmission electron micrograph (Figure 22b:M-NC11) was the same regardless of the cutting direction, it was concluded that M-NC11 consists of clay networks that form a large number of connected clay spheres $(100-300 \mathrm{~nm}$ in diameter), with a $20-\mathrm{nm}$ thick outer clay shell with the bulk of the flexible PMEA packed inside.

The clay network structure proposed is depicted graphically in Figure 24a. The following mechanism for its formation is suggested. As the hydrophobic PMEA chains tend to aggregate during the course of in situ polymerization, the primary clay platelets are squeezed out of these regions and form clay aggregates in which PMEA chains are attached to their surfaces. These clay aggregates and the bulk of the PMEA form nanometer-sized spherical structures consisting of an 


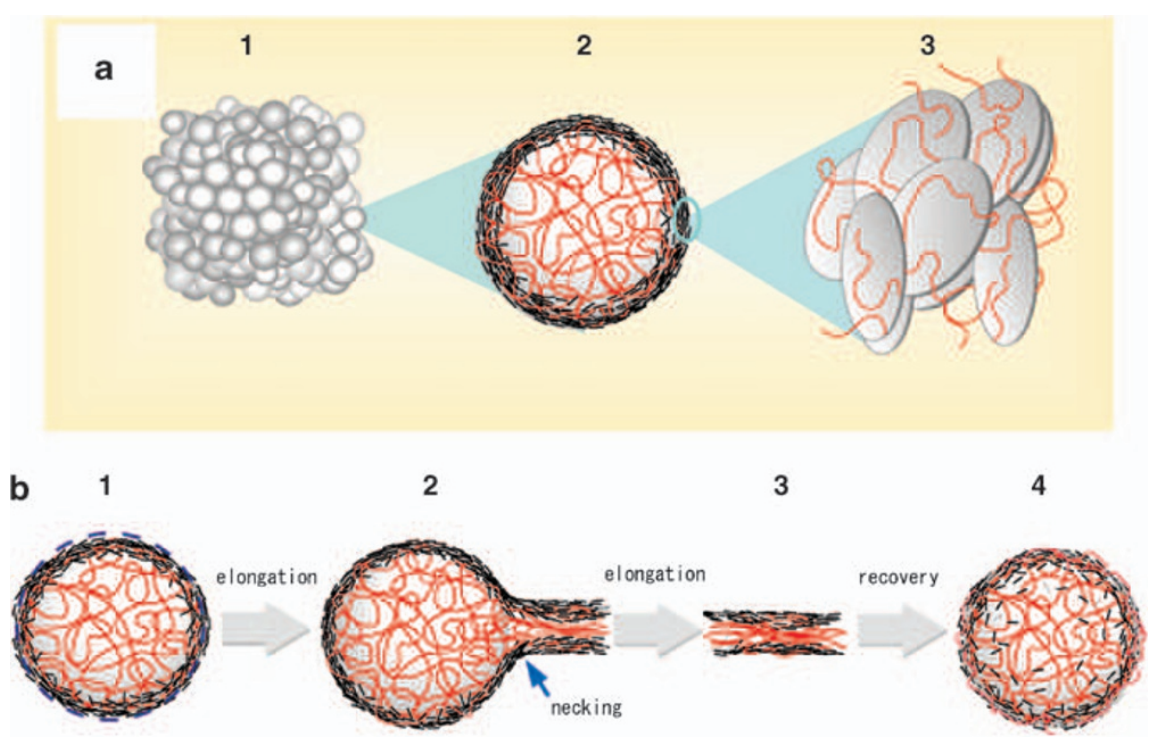

Figure 24 (a) Schematic illustrations of clay network morphology and (b) the deformation process for M-NC11. (a-1) Clay network structure. (a-2) A clay/ poly(2-methoxyethyl acrylate) (PMEA) sphere. (a-3) Clay outer shell. (b-1) Elastic deformation of the clay/PMEA sphere. (b-2) Deformation with necking point. (b-3) Further elongation over the $\mathrm{I}_{\mathrm{b}}$ point. (b-4) Structure after recovery from large elongation. ${ }^{11}$

outer shell of aggregated clay (Figure 24a(3)) and an inner PMEA core (Figure 24a(2)). Thus, a nanostructured morphology was latently formed in the preformed gel state; this nanostructure became apparent when water was removed. As each sphere was connected to neighboring spheres, a 3D clay network was formed (Figure 24a(1)). The sphere diameter varied with $C_{\text {clay }}$ and naturally decreased with an increase in $C_{\text {clay. }}$. When $C_{\text {clay }}$ was very low, a ribbon-like structure was formed instead of a spherical structure, as expected. Despite the formation of such structures, M-NCs are transparent because the clay-shell thickness is very small and the refractive indices of PMEA (1.47) and clay (1.50) are similar.

The characteristic tensile deformation of M-NCs (Figure 23a: I-III stages) is also explicable in terms of the proposed model for the clay network, as shown in Figure 24b. The extraordinarily high elongations (1000-3000\%) observed in M-NCs are attributable to two main factors. One is the highly contracted form of the randomly coiled PMEA chains, which is the result of syneresis during polymerization and shrinkage during the drying process. In the case of deswollen polymer networks, it has been reported that extensibility may increase considerably, reaching $\lambda_{\max }=18-30,{ }^{78}$ because of its supercoilded structure. The results for M-NCs are consistent with those obtained for supercoiled polymer chains. The other factor is the formation of a crosslinked structure between PMEA and clay. If there are no crosslinks to bear the applied stress, the contracted polymer chains can simply be drawn (at very low stress) by slipping. This has been observed in the case of M-LR. In the case of M-NCs, the supercoiled PMEA chains, the ends of which may interact with the exfoliated clay, undergo extensive elongation and may retract.

\section{CONCLUSION}

Using novel organic (polymer)/inorganic (clay) network structures, we created new types of soft polymeric materials, a NC gel and a soft $\mathrm{P}-\mathrm{NC}$ (M-NC), by in situ free-radical polymerization in the presence of clay nanoparticles in aqueous systems, in which exfoliated clay nanoplatelets function as multifunctional crosslinkers. Both NC gels and M-NCs had extraordinary optical, mechanical and stimulisensitive properties, in addition to a number of new characteristics.
All the properties and new characteristics can be attributed to each unique organic/inorganic network structure. NC gels and M-MCs can be prepared in various forms and sizes, and their properties can be controlled over a wide range by changing the compositions and their circumstances. Thus, NC gels expand the possibility of polymer hydrogels as environmentally friendly (water-based) materials, and M-NCs similarly as advanced nanocomposites with high inorganic contents. NC gels and M-NCs, which overcome the most serious disdavantages of conventional hydrogels and polymer nanocomposites, are promising soft polymeric materials that can be used in various fields including medical, biomedical, analytical and electronic devices.

\section{ACKNOWLEDGEMENTS}

I thank Kawamura Institute of Chemical Research and DIC for their support toward carrying out the works introduced in this review paper. I also thank collaborators, Mr T Takehisa, Dr H Li, Dr T Takada, Dr K Murata and other colleagues in my laboratory, Prof. M Shibayama and Dr GC Eastmond. I acknowledge the financial support provided by the Ministry of Education, Science, Sports and Culture, Japan (Grant-in-Aid for Scientific Research (B20350109 and C16550181), and by the New Energy and Industrial Technology Development Organization (NEDO) (Project 2006-2008), Japan.

1 Saegusa, T. \& Chujo, Y. Organic-inorganic polymer hybrids. Makromol. Chem. Macromol. Symp. 64, 1-9 (1992).

2 Giannelis, E. P. Polymer layered silicate nanocomposites. Adv. Mater. 8, 29-35 (1996).

3 Mark, J. E. New developments and directions in the area of elastomers and rubberlike elasticity. Macromol. Symp. 201, 77-83 (2003).

4 Okada, K. \& Usuki, A. Twenty years of polymer-clay nanocomposites. Macromol. Mater. Eng. 291, 1449-1476 (2006).

5 Usuki, A., Kojima, Y., Kawasumi, M., Okada, A., Fukushima, Y., Kurauchi, T. \& Kamigaito, O. Synthesis of nylon 6-clay hybrid. J. Mat. Res. 8, 1179-1184 (1993).

6 Haraguchi, K. \& Takehisa, T. Nanocomposite hydrogels: a unique organic-inorganic network structure with extraordinary mechanical, optical, and swelling/de-swelling properties. Adv. Mater. 14, 1120-1124 (2002).

7 Haraguchi, K. \& Li, H.- J. Control of the coil-to-globule transition and ultrahigh mechanical properties of PNIPA in nanocomposite hydrogels. Angew. Chem. Int. Ed. 44, 6500-6504 (2005). 
8 Haraguchi, K. Nanocomposite gels: new advanced functional soft materials. Macromol. Symp. 256, 120-130 (2007)

9 Haraguchi, K. Nanocomposite hydrogels. Curr. Opin. Solid State Mat. Sci. 11, 47-54 (2007).

10 Fukasawa, M., Sakai, T., Chung, U- I. \& Haraguchi, K. Synthesis and mechanical properties of a nanocomposite gel consisting of a tetra-peg/clay network. Macromolecules 43, 4370-4378 (2010).

11 Haraguchi, K., Ebato, M. \& Takehisa, T. Polymer-clay nanocomposites exhibiting abnormal necking phenomena accompanied by extremely large reversible elongations and excellent transparency. Adv. Mater. 18, 2250-2254 (2006).

12 Haraguchi, K., Masatoshi, S., Kotobuki, N. \& Murata, K. Thermoresponsible cell adhesion/detachment on transparent nanocomposite films consisting of poly(2-methoxyethyl acrylate) and clay. J. Biomater. Sci. Polym. Ed. doi:10.1163/ $092050610 \times 540459$ (2011)

13 Tanaka, T. Gels. Sci. Am. 244, 110-123 (1981).

14 Heskins, M. \& Guillet, J. E. Solution properties of poly(N-isopropylacrylamide). J. Macromol. Sci. Part A 2, 1441-1455 (1968).

15 Matsumoto, A., Yoshida, R. \& Kataoka, K. Glucose-responsive polymer gel bearing phenylborate derivative as a glucose-sensing moiety operating at the physiological $\mathrm{pH}$. Biomacromolecules 5, 1038-1045 (2004).

16 Cai, W., Anderson, E. C. \& Gupta, R. B. Separation of lignin from aqueous mixtures by ionic and nonionic temperature-sensitive hydrogels. Ind. Eng. Chem. Res. 40, 2283-2288 (2001).

17 Okano, T., Bae, Y. H., Jacobs, H. \& Kim, S. W. Thermally on-off switching polymers for drug permeation and release. J. Control Release 11, 255-265 (1990).

18 Stayton, P. S., Shimoboji, T., Long, C., Chilkoti, A., Chen, G., Harris, J. M. \& Hoffman, A. S. Control of protein-ligand recognition using a stimuli-responsive polymer. Nature 378, 472-474 (1995).

19 Yamato, M. \& Okano, T. Cell sheet engineering. Mater. Today 7, 42-47 (2004).

20 Haraguchi, K., Takehisa, T. \& Fan, S. Effects of clay content on the properties of nanocomposite hydrogels composed of poly ( $\mathrm{N}$-isopropylacrylamide) and clay. Macromolecules 35, 10162-10171 (2002).

$21 \mathrm{Xu}, \mathrm{Y} ., \mathrm{Li}, \mathrm{G}$. \& Haraguchi, K. Gel formation and molecular characteristics of poly ( $\mathrm{N}$-isopropylacrylamide)prepared by free-radical redox polymerization in aqueous solution. Macromol. Chem. Phys. 211, 977-987 (2010).

22 Okumura, Y. \& Ito, K. The polyrotaxane gel: a topological gel by figure-of-eight crosslinks. Adv. Mater. 13, 485-487 (2001).

23 Gong, J. P., Katsuyama, Y., Kurokawa, T. \& Osada, Y. Double-network hydrogels with extremely high mechanical strength. Adv. Mater. 15, 1155-1158 (2003).

24 Huang, T., Hongguang, X., Jiao, K., Zhu, L., Brown, H. R. \& Wang, H. A novel hydrogel with high mechanical strength: a macromolecular microsphere composite hydrogel. Adv. Mater. 19, 1622-1626 (2007).

25 Sakai, T., Matsunaga, T., Yamamoto, Y., Ito, C., Yoshida, R., Suzuki, S., Sasaki, N., Shibayama, M. \& Chung, U. I. Design and fabrication of a high-strength hydrogel with ideally homogeneous network structure from tetrahedron-like macromonomers. Macromolecules 41, 5379-5384 (2008).

26 Haraguchi, K., Farnworth, R., Ohbayashi, A. \& Takehisa, T. Compositional effects on mechanical properties of nanocomposite hydrogels composed of poly ( $\mathrm{N}, \mathrm{N}$-dimethylacrylamide) and clay. Macromolecules 36, 5732-5741 (2003).

27 Haraguchi, K. \& Li, H.- J. Mechanical properties and structure of polymerclay nanocomposite gels with high clay content. Macromolecules 39, 1898-1905 (2006).

28 Rosta, L. \& Gunten, H. R. von. Light scattering characterization of laporite sols. J. Colloid Interface Sci. 134, 397-406 (1990).

29 Haraguchi, K. \& Song, L. Microstructures formed in co-cross-linked networks and their relationships to the optical and mechanical properties of PNIPA/clay nanocomposite gels. Macromolecules 40, 5526-5536 (2007).

30 Haraguchi, K. \& Takada, T. Synthesis and characteristics of nanocomposite gels prepared by in situ photopolymerization in an aqueous system. Macromolecules $\mathbf{4 3}$ 4294-4299 (2010).

31 Haraguchi, K., Li, H.- J., Matsuda, K., Takehisa, T. \& Elliot, E. Mechanism of forming organic/inorganic network structures during in-situ free-radical polymerezation in PNIPA-clay nanocomposite hydrogels. Macromolecules 38, 3482-3490 (2005).

32 Shibayama, M., Suda, J., Karino, T., Okabe, S., Takehisa, T. \& Haraguchi, K. Structure and dynamics of poly ( $\mathrm{N}$-isopropylacrylamide)-clay nanocomposite gels. Macromolecules 37, 9606-9612 (2004).

33 Miyazaki, S., Karino, T., Endo, H., Haraguchi, K. \& Shibayama, M. Clay concentration dependence of microstructure in deformed poly( $\mathrm{N}$-isopropylacrylamide)-clay nanocomposite gels. Macromolecules 39, 8112-8120 (2006).

34 Miyazaki, S., Endo, H., Karino, T., Haraguchi, K. \& Shibayama, M. Gelation mechanism of poly ( $\mathrm{N}$-isopropyl acrylamide)-clay nanocomposite gels. Macromolecules 40, 4287-4295 (2007).

$35 \mathrm{Nie}$, J., Du, B. \& Oppermann, W. Dynamic fluctuations and spatial inhomogeneities in poly(N- isopropylacrylamide)/clay nanocomposite hydrogels studied by dynamic light scattering. J. Phys. Chem. B 110, 11167-11175 (2006).

36 Haraguchi, K. \& Li, H.- J. The effect of water content on the ultimate properties of rubbery nanocomposite gels. J. Polym. Sci. Part B Polym. Phys. 47, 2328-2340 (2009).

37 Haraguchi, K., Xu, Y. \& Li, G. Molecular characteristics of poly(N-isopropylacrylamide) separated from nanocomposite gels by removal of clay from the polymer/clay network. Macromol. Rapid. Commun. 31, 718-723 (2010).

38 Tobolsky, A. V., Carlson, D. W. \& Indictor, N. Rubber elasticity and chain configuration. J. Polym. Sci. 54, 175-192 (1961).

39 Flory, P. J. \& Rehner, J. Jr Statistical mechanics of cross-linked polymer networks II. Swelling. J. Chem. Phys. 11, 521-526 (1943).
40 Haraguchi, K., Li, H.- J., Song, L. \& Murata, K. Tunable optical and swelling/deswelling properties associated with control of the coil-to-globule transition of poly ( $\mathrm{N}$-isopropylacrylamide) in polymer-clay. Macromolecules 40, 6973-6980 (2007).

41 Baker, J. P., Hong, L. H., Blanch, H. W. \& Prausnitz, J. M. Effect of initial total monomer concentration on the swelling behavior of cationic acrylamide-based hydrogels. Macromolecules 27, 1446-1454 (1994).

42 Dijkstra, M., Hansen, J. P. \& Madden, P. A. Gelation of a clay colloid suspension. Phys. Rev. Lett. 75, 2236-2239 (1995).

43 Haraguchi, K. \& Li, H.- J. Mechanical properties of nanocomposite hydrogels consisting of organic/inorganic networks and the effects of clay modification thereto. J. Network Polym. Jpn. 25, 2-12 (2004).

44 Haraguchi, K., Li, H.-J., Ren, H. \& Zhu, M. Modification of nanocomposite gels by irreversible rearrangement of polymer/clay network structure through drying. Macromolecules 43, 9848-9853 (2010).

45 Smith, T. L. \& Stedry, P. J. Time and temperature dependence of the ultimate properties of an SBR rubber at constant elongations. J. Appl. Phys. 31, 1892-1898 (1960).

46 Zhang, X. \& Zhuo, R. Dynamic properties of temperature-sensitive poly ( $\mathrm{N}$-isopropylacrylamide) gel cross-linked through siloxane linkage. Langmuir 17, 12-16 (2001).

47 Okajima, T., Harada, I., Nishio, K. \& Hirotsu, S. Discontinuous crossover between fast and slow kinetics at the volume phase transition in poly- $\mathrm{N}$-isopropylacrylamide gels. Jpn. J. Appl. Phys. 39, L875-L877 (2000).

48 Yoshida, R., Uchida, K., Kaneko, Y., Sakai, K., Kikuchi, A., Sakurai, Y. \& Okano, T. Comb-type grafted hydrogels with rapid de-swelling response to temperature changes. Nature 374, 240-242 (1995).

49 Tanaka, T. \& Fillmore, D. J Kinetics of swelling of gels. J. Chem. Phys. 70, 1214-1218 (1979).

50 Haraguchi, K., Li, H.- J. \& Song, L. The unique optical and physical properties of soft, transparent, stimulus-sensitive nanocomposite gels. Proc. SPIE 6654, 665400 1-11 (2007).

51 Song, L., Zhu, M., Chen, Y. \& Haraguchi, K. Temperature- and pH-sensitive nanocomposite gels with semi-interpenetrating organic/inorganic networks. Macromol. Chem. Phys. 209, 1564-1575 (2008).

52 Urayama, K. Selected issues in liquid crystal elastomers and gels. Macromolucules 40, 2277-2288 (2007).

53 Yokoyama, F., Achife, E. C., Matsuoka, M., Shimamura, K., Yamashita, Y. \& Monobe, K. Morphology of oriented calcium alginate gels obtained by the flow-gelation method. Polymer 32, 2911-2916 (1991).

54 Stellwagen, J. \& Stellwagen, N. C. Orientation of the agarose gel matrix in pulsed electric fields. Nucleic Acids Res. 17, 1537-1548 (1989).

55 Murata, K. \& Haraguchi, K. Optical anisotropy in polymer-clay nanocomposite hydrogel and its change on uniaxial deformation. J. Mater. Chem. 17, 3385-3388 (2007).

56 Nishida, T., Endo, H., Osaka, N., Li, H.- J., Haraguchi, K. \& Shibayama, M. Deformation mechanism of nanocomposite gels studied by contrast variation small-angle neutron scattering. Phys. Rev. E. 80, 030801(R) 1-4 (2009).

57 Haraguchi, K. \& Takada, T. Characteristic sliding frictional behavior on the surface of nanocomposite hydrogels consisting of organic-inorganic network structure. Macromol. Chem. Phys. 206, 1530-1540 (2005).

58 Zhang, J., Pelton, R. \& Deng, Y. Temperature-dependent contact angles of water on poly(N-isopropylacrylamide) gels. Langmuir 11, 2301-2302 (1995).

59 Teare, D. O. H., Barwick, D. C., Schofield, W. C. E., Garrod, R. P., Beeby, A. \& Badyal, J. P. S. Functionalization of solid surfaces with thermoresponsive protein-resistant films. J. Phys. Chem. B 109, 22407-22412 (2005).

60 Sun, T., Wang, G., Feng, L., Liu, B., Ma, Y., Jiang, L. \& Zhu, D. Reversible switching between superhydrophilicity and superhydrophobicity. Angew. Chem. Int. Ed. 43, 357-360 (2004)

61 Haraguchi, K., Li, H.- J. \& Okumura, N. Hydrogels with hydrophobic surfaces: abnormally high contact angles for water on pnipa nanocomposite hydrogels. Macromolecules 40, 2299-2302 (2007); Research Highlights. Nature 446, 350 (2007).

62 Haraguchi, K., Li, H.- J. \& Song, L. Unusually high hydrophobicity and its changes observed on the newly-created surfaces of pnipa/clay nanocomposite hydrogels. J. Colloid Interface Sci. 326, 41-50 (2008).

63 Takizawa, T., Mori, Y. \& Yoshizato, K. Cell culture on a thermoresponsive polymer surface. Biotechnology 8, 854-856 (1990).

64 Akiyama, Y., Kikuchi, A., Yamato, M. \& Okano, T. Ultrathin poly(N-isopropylacrylamide) grafted layer on polystyrene surfaces for cell adhesion/detachment control. Langmuir 20, 5506-5511 (2004).

65 Haraguchi, K., Takehisa, T. \& Ebato, M. Control of cell cultivation and cell sheet detachment on the surface of polymer/clay nanocomposite hydrogels. Biomacromolecules 7, 3267-3275 (2006).

66 Haraguchi, K. \& Matsuda, M. Spontaneous formation of characteristic layered morphologies in porous nanocomposites prepared from nanocomposite hydrogels. Chem. Mater. 17, 931-934 (2005)

67 Haraguchi, K., Taniguchi, S. \& Takehisa, T. Reversible force generation in a temperature-responsive nanocomposite hydrogel consisting of poly ( $\mathrm{N}$-isopropylacrylamide) and clay. Chem. Phys. Chem. 6, 238-241 (2005).

68 Song, L., Zhu, M., Chen, Y. \& Haraguchi, K. Surface-patterning of nanocomposite hydrogel film by direct replica molding and subsequent change in pattern size. Polym. J. 40, 800-805 (2008).

69 Haraguchi, K. \& Takehisa, T. Novel manufacturing process of nanocomposite hydrogel for bio-applications. Proc. IMECE (ASME) 2005, IMECE 80533 1-8 (2005). 
70 Gilman, J. W., Jackson, C. L., Morgan, A. B., Harris, R., Manias, J. E., Giannelis, E. P., Wuthenow, M., Hilton, D. \& Phillips, S. H. Flammability properties of polymer-layered-silicate nanocomposites. polypropylene and polystyrene nanocomposites. Chem. Mater. 12, 1866-1873 (2000).

71 Idemura, S. \& Haraguchi, K. Glass-polyamide composite and process for producing the same (2000) USP6063862.

72 Goda, H. \& Higashino, T. Epoxy resin composition and process for producing silanemodified epoxy resin (2003) USP6525160.

73 Haraguchi, K., Ebato, M. \& Takehisa, T. Stretchy clay composites. Science 314, 19 (2006).

74 Tanaka, M., Motomura, T., Ishii, N., Shimura, K., Onishi, M., Mochizuki, A. \& Hatakeyama, T. Cold crystallization of water in hydrated poly(2-methoxyethyl acrylate) (PMEA). Polym. Int. 49, 1709-1713 (2000).
75 Saito, N., Motoyama, S. \& Sawamoto, J. Effects of new polymer-coated extracorporeal circuits on biocompatibility during cardiopulmonary bypass. Artif. Organs 24, 547-554 (2000).

76 Baykut, D., Bernet, F., Wehrle, J., Weichelt, K., Schwartz, P. \& Zerkowski, H.- R. New surface biopolymers for oxygenators: an in vitro hemocompatibility test of poly(2-methoxyethylacrylate). Eur. J. Med. Res. 6, 297-305 (2001).

77 Tanaka, M., Mochizuki, A., Ishii, N., Motomura, T. \& Hatakeyama, T. Study of blood compatibility with poly(2-methoxyethyl acrylate). Relationship between water structure and platelet compatibility in poly(2-methoxyethylacrylate-co-2-hydroxyethylmethacrylate). Biomacromolecules 3, 36-41 (2002).

78 Urayama, K. \& Kohjiya, S. Extensive stretch of polysiloxane network chains with random- and super-coiled conformations. Eur. Phys. J. B-2, 75-78 (1998).

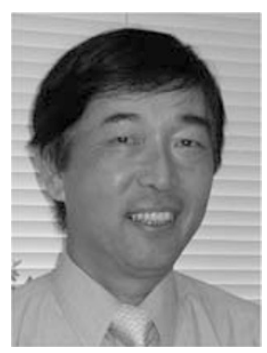

Kazutoshi Harguchi was born in November 1950 in Saga, Japan. He graduated from the Department of Applied Chemistry, Faculty of Engineering, Kyushu University in 1973, and received his $\mathrm{PhD}$ from Kyushu University in 1978 under the supervision of Prof. Motowo Takayanagi. He joined the Kawamura Institute of Chemical Research (KICR) in 1978 and has continued in research to the present day. Meanwhile, he worked in Liverpool University, UK, as a post-doctoral fellow in 1979-1981. Also, he worked in Dainippon Ink and Chemical (now DIC) from 1985 to 1999 as a project manager. He became a director of KICR in 2001, and a general manager (director) in 2006. His major research fields are polymer physics and synthesis of carbon fiber, CF composites, organic/inorganic nanocomposites and polymer hydrogels. He received the Award of the Society of Fiber Science and Technology, Japan, in 2003, and the Award of the Society of Polymer Science, Japan in 2010. 